\title{
Retrospective analysis of arterial occlusive events in the PACE trial by an independent adjudication committee
}

\author{
James L. Januzzi ${ }^{*}$ (D), Joseph M. Garasic ${ }^{1}$, Scott E. Kasner², Vickie McDonald ${ }^{3}$, Mark C. Petrie ${ }^{4}$, Jonathan Seltzer ${ }^{5}$, \\ Michael Mauro ${ }^{6}$, Kevin Croce ${ }^{7}$, Ellin Berman ${ }^{6}$, Michael Deininger ${ }^{8}$, Andreas Hochhaus ${ }^{9}$, Javier Pinilla-lbarz ${ }^{10}$, \\ Franck Nicolini ${ }^{11}$, Dong-Wook Kim ${ }^{12}$, Daniel J. DeAngelo ${ }^{13}$, Hagop Kantarjian ${ }^{14}$, Jing Xu ${ }^{15}$, Tracey Hall ${ }^{15}$, \\ Shouryadeep Srivastava ${ }^{15}$, Daniel Naranjo ${ }^{15}$ and Jorge Cortes ${ }^{16}$
}

\begin{abstract}
Background: The phase 2 PACE (Ponatinib Ph+ ALL and CML Evaluation) trial of ponatinib showed robust longterm benefit in relapsed Philadelphia chromosome-positive $(\mathrm{Ph}+)$ leukemia; arterial occlusive events (AOEs) occurred in $\geq 25 \%$ of patients based on investigator reporting. However, AOE rates vary depending on the definitions and reporting approach used.

Methods: To better understand clinically relevant AOEs with ponatinib, an independent cardiovascular adjudication committee reviewed 5-year AOE data from the PACE trial according to a charter-defined process and standardized event definitions.
\end{abstract}

Results: A total of 449 patients with chronic myeloid leukemia (CML) or Ph+ acute lymphoblastic leukemia (ALL) received ponatinib (median age 59 y; $47 \%$ female; $93 \% \geq 2$ prior tyrosine kinase inhibitors (TKIs); median follow-up, 37.3 months). The adjudicated AOE rate (17\%) was lower than the non-adjudicated rate (i.e., rate before adjudication; $25 \%)$. The only adjudicated AOE in $>2 \%$ of patients was peripheral arterial occlusive disease (4\%). Exposure-adjusted incidence of newly occurring adjudicated AOEs decreased over time. Patients with multiple baseline cardiovascular risk factors had higher adjudicated $A O E$ rates than those without risk factors.

Conclusions: This independent adjudication study identified lower AOE rates than previously reported, suggesting earlier overestimation that may inaccurately reflect AOE risk with ponatinib. This trial was registered under ClinicalTrials.gov identifier NCT01207440 on September 23, 2010 (https://clinicaltrials.gov/ct2/show/NCT01207440).

Keywords: Acute lymphoblastic leukemia, Chronic myeloid leukemia, Safety, Tyrosine kinase inhibitor

\section{Background}

Ponatinib, a pan-BCR::ABL1 inhibitor, is an orally active third-generation tyrosine kinase inhibitor (TKI) designed to potently inhibit BCR::ABL1 with or without any point mutation, including $B C R:: A B L 1^{T 315 I}[1]$. In the

\footnotetext{
*Correspondence: JJANUZZI@PARTNERS.ORG

${ }^{1}$ Massachusetts General Hospital, 55 Fruit Street, Boston, MA, USA

Full list of author information is available at the end of the article
}

pivotal phase 2 PACE (Ponatinib Ph+ ALL and CML Evaluation) trial, ponatinib demonstrated robust clinical activity with rapid, deep, and long-term responses, progression-free survival (PFS), and overall survival in patients with chronic-phase chronic myeloid leukemia (CP-CML), $\geq 90 \%$ of whom had failed treatment with $\geq 2$ TKIs, regardless of the presence or absence of BCR::ABL1 mutations, including T315I $[2,3]$. The 5-year results of the PACE trial confirmed the durability original author(s) and the source, provide a link to the Creative Commons licence, and indicate if changes were made. The images or other third party material in this article are included in the article's Creative Commons licence, unless indicated otherwise in a credit line to the material. If material is not included in the article's Creative Commons licence and your intended use is not permitted by statutory regulation or exceeds the permitted use, you will need to obtain permission directly from the copyright holder. To view a copy of this licence, visit http://creativecommons.org/licenses/by/4.0/. The Creative Commons Public Domain Dedication waiver (http://creativeco mmons.org/publicdomain/zero/1.0/) applies to the data made available in this article, unless otherwise stated in a credit line to the data. 
of these responses with a 5-year overall survival rate of $73 \%$ for CP-CML [3]. However, arterial occlusive events (AOEs) were reported by investigators in $25 \%$ in the overall population (serious AOEs, $20 \%$ ) and $31 \%$ in the CP-CML population (serious AOEs, 26\%) in the 5-year follow-up [3]. The exposure-adjusted incidence of newly occurring AOEs decreased from year 1 (15.8 patients with events per 100 patient-years in the total population) to year 5 (3.9 per 100 patient-years) [3]. The incidence of AOEs associated with ponatinib use has varied widely in subsequent reports. Two retrospective studies have reported an absence or very low incidence (6\%) of AOEs [4, 5]. Other real-world studies have reported AOE rates ranging from 18 to $26 \%[6,7]$. Multiple factors may contribute to variability in reported $\mathrm{AOE}$ rates, including differences in patient populations, as well as differences in the clinical definitions used to identify and categorize vascular occlusive events. One of the most important factors is the lack of a standardized approach for defining and capturing AOEs with BCR::ABL1 TKIs.

The AOE incidence rate reported for PACE was based on a list of approximately 400 Medical Dictionary for Regulatory Activities (MedDRA) preferred terms developed by the sponsor. However, differences in the preferred terms used to define AOEs led to variability in AOE incidence rates. Some preferred terms included in the AOE analysis of PACE are highly sensitive for identification of potential AOEs but may not themselves indicate the occurrence of arterial occlusions, frequently including symptoms or descriptions rather than events; these include chest pain, cold hands, dysarthria, and poor peripheral circulation. This approach to characterize AOEs based on adverse event terms results in broadly capturing non-specific symptoms that may be associated with AOE rather than true AOEs and may thus overestimate the incidence of clinically meaningful events.

A clear understanding of clinically relevant $\mathrm{AOE}$ risk is imperative when characterizing the benefit-risk profile of ponatinib. Patients with CP-CML who become resistant to a second-generation BCR::ABL1 TKI, either with or without a $B C R:: A B L 1$ gene mutation, generally experience low response rates and poor survival if treated with another second-generation TKI [8, 9]. Importantly, ponatinib is the only currently available TKI effective in patients with the $B C R:: A B L 1^{T 315 I}$ mutation [3]. Therefore, the potential for improved survival and duration of response on ponatinib may outweigh the risk of AOEs [8, 9]. However, the lack of clear data regarding clinically meaningful AOEs has led to confusion about how to optimally use ponatinib to treat relapsed/refractory CML and Philadelphia chromosome-positive $(\mathrm{Ph}+)$ acute lymphoblastic leukemia (ALL) and, in some instances, avoidance in patients who could potentially benefit. To provide a more accurate characterization of AOE incidence with ponatinib, an independent adjudication committee of experts was convened to retrospectively adjudicate all AOE reports in the PACE trial in a standardized, rigorous manner.

\section{Methods \\ PACE trial design}

The phase 2 PACE trial (ClinicalTrials.gov identifier: NCT01207440) enrolled adults with CML or Ph+ ALL whose disease was resistant or intolerant to dasatinib or nilotinib, or who had the $B C R:: A B L 1^{T 315 I}$ mutation regardless of prior TKI use [3]. All patients received ponatinib at a starting dose of $45 \mathrm{mg}$ once daily (qd); dose reductions to 30 or $15 \mathrm{mg}$ qd were applied per protocol (Table 1) to manage adverse events (AEs), or implemented proactively following recommendations from the sponsor in October 2013 in response to AOEs emerging as notable AEs. The trial has been completed; detailed methods are published [2, 3].

\section{Adjudication methods}

All activities related to the adjudication of AOEs were conducted by ACI Clinical (Bala Cynwyd, PA), including the identification of an independent adjudication committee. ACI Clinical is a clinical research organization with expertise in Endpoint Adjudication and Data Monitoring Committees to support safety decisions around clinical development programs. ACI Clinical was contracted by the sponsor; adjudication activities were not part of the PACE trial.

\section{Identification of AEs for adjudication}

To ensure all relevant potential events were captured, the PACE AE dataset (449 patients with 12,224 AE records; extraction date: May 9, 2018) was searched using a comprehensive set of 604 preferred terms potentially relevant to AOEs that was developed by the sponsor (Table 2). This search strategy, which was more comprehensive than that used in initial analyses of the PACE trial, identified 181 patients and 455 $\mathrm{AE}$ records for adjudication (Fig. 1A). In addition, all patient deaths not attributable to disease progression by the clinical investigator were reviewed by the chair of the adjudication committee (described below) for identification of potential fatal AOEs. The adjudication committee identified 45 fatal events for review. In total, 202 patients and 490 events were submitted 
to the independent adjudication committee for review (Fig. 1A).

An individual case package containing all available clinical information (including medical history) was created for each event and provided to the adjudication committee members for their review. If a patient experienced more than 1 event within $48 \mathrm{~h}$, these events were adjudicated as potentially representing a single clinical event, unless the case evidence suggested they were independent events. Individual events occurring $>48 \mathrm{~h}$ apart were adjudicated as independent events. All data were from the clinical trial database that was in SAS format and structured in conformance to CDISC SDTM format; no other source material was available.

\section{Adjudication procedure}

An adjudication committee of academic research clinicians who are highly experienced in adjudication activities in cardiovascular trials was appointed by ACI Clinical. The adjudication committee of 5 independent academic experts ( 3 cardiologists, 1 vascular medicine specialist, and 1 vascular neurologist) retrospectively adjudicated suspected cases of arterial occlusive events in the PACE study. The committee followed a predefined process outlined in the adjudication charter developed by ACI clinical. The charter defined the responsibilities of the adjudication committee and the adjudication endpoints using established definitions developed by the 2014 American College of Cardiology (ACC)/American Heart Association (AHA) guideline [10], and the definitions for cardiovascular and stroke outcomes developed by the Standardized Data Collection for Cardiovascular Trials Initiative (SCTI) and the US Food and Drug Administration [11, 12]. All suspected AOEs identified in the PT search were assessed using the charter definitions (Table 3) for myocardial infarction; heart failure if attributed to an AOE, which may include coronary artery disease, arterial hypertension, cardiomyopathy, or myocardial infarction; hospitalization for unstable angina; stroke and other cerebrovascular events; and peripheral vascular disease. Any events meeting the criteria of these endpoints were considered adjudicated AOEs. Specific criteria were required (e.g., revascularization, change in cardiac biomarkers, diagnostic evidence as shown by computerized tomography scan, magnetic resonance imaging, etc.) to determine the presence of a clinical endpoint. The adjudication committee members were blind to ponatinib dose at the time of the event, whether dose modifications were made, and the investigator's opinion on AE causality.

During the adjudication process, the committee reviewed all potential AOEs, as well as any AEs identified in a Cardiac Failure Standard MedDRA Query (SMQ), to determine whether any heart failure events were AOEs. Two members of the adjudication committee independently evaluated whether an individual case met the prespecified event definitions (Fig. 1B). If agreement between 2 members was not reached for cases of AOEs or heart failure, the case was reviewed by a third cardiologist adjudication committee member; if agreement was not reached with 3 votes, the case was reviewed at a panel meeting. If agreement was not reached for cases of stroke, deep vein thrombosis, pulmonary embolism, and peripheral vascular disease, the case was discussed at a panel meeting with the appropriate neurologist and/ or vascular specialist member(s). All fatal events were decided by consensus of adjudicators.

Events that met one of the charter-defined endpoint definitions were further categorized depending on the event type (e.g., myocardial infarction, peripheral arterial occlusive disease, deep vein thrombosis, etc.). Nonadjudicated AOEs that were recorded as symptoms (e.g.,

Table 1 Dose reduction recommendations (as of 2013)

Dose reduction recommendations

In October 2013, the following specific recommendations were formulated after discussions with the US FDA on evolving observations of arterial occlusive events in patients treated with ponatinib:

All chronic phase chronic myeloid leukemia (CP-CML) patients on study who already had achieved major cytogenetic response (MCyR) should have had their dose reduced to $15 \mathrm{mg}$ daily, unless, in the judgment of the investigator, the benefit/risk analysis, taking into account the patient's disease characteristics, BCR::ABL mutation status, and the patient's cardiovascular risk justified treatment with a higher dose

All CP-CML patients on study who had not yet achieved MCyR should have had their dose reduced to $30 \mathrm{mg}$ daily, unless, in the judgment of the investigator, the benefit/risk analysis, taking into account the patient's disease characteristics, $B C R:: A B L$ mutation status, and the patient's cardiovascular risk justified treatment with a higher dose

All acute phase chronic myeloid leukemia (AP-CML), blast phase chronic myeloid leukemia (BP-CML), and Ph+ acute lymphoblastic leukemia (ALL) patients on study should have had their dose reduced to $30 \mathrm{mg}$ daily, unless, in the judgment of the investigator, the benefit/risk analysis, taking into account the patient's disease characteristics, $B C R:: A B L$ mutation status, and the patient's cardiovascular risk justified treatment with a higher dose

All patients who lost response at a lower dose may have their dose escalated (up to a maximum of $45 \mathrm{mg}$ daily) as long as the dose was not lowered as a result of an adverse event (AE) 
Table 2 List of 604 preferred terms used to identify AEs for adjudication

\section{Preferred term (MEdDRA 21.0)}

Acute aortic syndrome

Acute coronary syndrome

Acute myocardial infarction

Administration site thrombosis

Adrenal thrombosis

\section{Agnosia}

Amaurosis

Amaurosis fugax

Amputation

Angina pectoris

Angina unstable

Anginal equivalent

Angiogram abnormal

Angiogram cerebral abnormal

Angiogram peripheral abnormal

Angioplasty

Angiosclerosis

Anterior segment ischaemia

Aortic arteriosclerosis

Aortic bypass

Aortic embolus

Aortic occlusion

Aortic restenosis

Aortic stenosis

Aortic surgery

Aortic thrombosis

Aortogram abnormal

Aphasia

Application site thrombosis

Arm amputation

Arterectomy

Arterectomy with graft replacement

Arterial bypass occlusion

Arterial bypass operation

Arterial bypass stenosis

Arterial bypass thrombosis

Arterial disorder

Arterial graft

Arterial insufficiency

Arterial occlusive disease

Arterial restenosis

Arterial stenosis

Arterial stent insertion

Arterial therapeutic procedure

Arterial thrombosis

Arteriogram abnormal

Arteriogram carotid abnormal

Arteriogram coronary abnormal

Arteriogram renal abnormal

\section{Diplegia}

Directional Doppler flow tests abnormal

Dissecting coronary artery aneurysm

Disseminated intravascular coagulation

Disseminated intravascular coagulation in newborn

Dry gangrene

Dysarthria

ECG electrically inactive area

ECG signs of myocardial infarction

ECG signs of myocardial ischaemia

Electrocardiogram Q wave abnormal

Electrocardiogram ST segment abnormal

Electrocardiogram ST segment depression

Electrocardiogram ST segment elevation

Electrocardiogram ST-T segment abnormal

Electrocardiogram ST-T segment depression

Electrocardiogram ST-T segment elevation

Electrocardiogram T wave abnormal

Electrocardiogram T wave inversion

Electrocardiogram $U$ wave inversion

Embolia cutis medicamentosa

Embolic cerebral infarction

Embolic pneumonia

Embolic stroke

Embolism

Embolism arterial

Embolism venous

Endarterectomy

Exercise electrocardiogram abnormal

Exercise test abnormal

External counterpulsation

Extremity necrosis

Extrinsic iliac vein compression

Femoral artery embolism

Finger amputation

Foetal cerebrovascular disorder

Foot amputation

Gangrene

Gastrointestinal ischaemia

Glomerular vascular disorder

Graft ischaemia

Graft thrombosis

Haemorrhage coronary artery

Haemorrhagic adrenal infarction

Haemorrhagic cerebral infarction

Haemorrhagic infarction

Haemorrhagic stroke

Haemorrhagic transformation stroke

Haemorrhagic vasculitis
Pituitary infarction

Placental infarction

Pneumatic compression therapy

Poor peripheral circulation

Popliteal artery entrapment syndrome

Portal shunt procedure

Portal vein cavernous transformation

Portal vein occlusion

Portal vein stenosis

Portal vein thrombosis

Portosplenomesenteric venous thrombosis

Post angioplasty restenosis

Post cardiac arrest syndrome

Post procedural myocardial infarction

Post procedural pulmonary embolism

Post procedural stroke

Post stroke depression

Post thrombotic syndrome

Posthaemorrhagic hydrocephalus

Postinfarction angina

Postoperative thrombosis

Postpartum thrombosis

Postpartum venous thrombosis

Precerebral arteriosclerosis

Precerebral artery occlusion

Precerebral artery thrombosis

Prinzmetal angina

Profundaplasty

Prosthetic vessel implantation

Pulmonary artery occlusion

Pulmonary artery stenosis

Pulmonary artery therapeutic procedure

Pulmonary artery thrombosis

Pulmonary embolism

Pulmonary endarterectomy

Pulmonary infarction

Pulmonary microemboli

Pulmonary thrombosis

Pulmonary tumour thrombotic microangiopathy

Pulmonary vein occlusion

Pulmonary vein stenosis

Pulmonary veno-occlusive disease

Pulmonary venous thrombosis

Quadriparesis

Quadriplegia

Raynaud's phenomenon

Renal arteriosclerosis

Renal artery angioplasty

Renal artery arteriosclerosis 
Table 2 (continued)

\section{Preferred term (MEdDRA 21.0)}

Arteriosclerosis

Arteriosclerosis coronary artery

Arteriosclerosis Monckeberg type

Arteriosclerotic gangrene

Arteriosclerotic retinopathy

Arteriospasm coronary

Arteriotomy

Arteriovenous fistula occlusion

Arteriovenous fistula thrombosis

Arteriovenous graft site stenosis

Arteriovenous graft thrombosis

Arteritis

Artificial blood vessel occlusion

Atherectomy

Atherosclerotic plaque rupture

Atrial appendage closure

Atrial thrombosis

Axillary vein thrombosis

Balint's syndrome

Basal ganglia infarction

Basal ganglia stroke

Basilar artery occlusion

Basilar artery stenosis

Basilar artery thrombosis

Biliary ischaemia

Blindness transient

Blood creatine phosphokinase abnormal

Blood creatine phosphokinase increased

Blood creatine phosphokinase MB abnormal

Blood creatine phosphokinase MB increased

Bone infarction

Bone marrow ischaemia

Brachial artery entrapment syndrome

Brachiocephalic arteriosclerosis

Brachiocephalic artery occlusion

Brachiocephalic artery stenosis

Brachiocephalic vein occlusion

Brachiocephalic vein stenosis

Brachiocephalic vein thrombosis

Brain hypoxia

Brain stem embolism

Brain stem infarction

Brain stem ischaemia

Brain stem stroke

Brain stem thrombosis

Budd-Chiari syndrome

Capsular warning syndrome

Cardiac arrest

Cardiac discomfort
Haemorrhoids thrombosed

Hand amputation

Hemianaesthesia

Hemiparesis

Hemiplegia

Heparin-induced thrombocytopenia

Hepatic artery embolism

Hepatic artery occlusion

Hepatic artery stenosis

Hepatic artery thrombosis

Hepatic infarction

Hepatic ischaemia

Hepatic vascular thrombosis

Hepatic vein embolism

Hepatic vein occlusion

Hepatic vein stenosis

Hepatic vein thrombosis

Homans'sign positive

Hypothenar hammer syndrome

Hypoxic-ischaemic encephalopathy

Iliac artery disease

Iliac artery embolism

lliac artery occlusion

lliac vein occlusion

Implant site thrombosis

Incision site vessel occlusion

Infarction

Inferior vena cava syndrome

Inferior vena caval occlusion

Infusion site thrombosis

Injection site thrombosis

Inner ear infarction

Instillation site thrombosis

Intermittent claudication

Interscapulothoracic amputation

Intestinal infarction

Intestinal ischaemia

Intra-aortic balloon placement

Intracardiac mass

Intracardiac thrombus

Intracranial artery dissection

Intracranial venous sinus thrombosis

Intraoperative cerebral artery occlusion

Ischaemia

Ischaemic cardiomyopathy

Ischaemic cerebral infarction

Ischaemic contracture of the left ventricle

Ischaemic enteritis

Ischaemic gastritis

\author{
Renal artery occlusion \\ Renal artery stenosis \\ Renal artery thrombosis \\ Renal embolism \\ Renal infarct \\ Renal ischaemia \\ Renal vascular thrombosis \\ Renal vein embolism \\ Renal vein occlusion \\ Renal vein thrombosis \\ Retinal artery embolism \\ Retinal artery occlusion \\ Retinal artery stenosis \\ Retinal artery thrombosis \\ Retinal infarction \\ Retinal ischaemia \\ Retinal vascular disorder \\ Retinal vascular occlusion \\ Retinal vascular thrombosis \\ Retinal vein occlusion
}

Retinal vein thrombosis

Reversible cerebral vasoconstriction syndrome

Reversible ischaemic neurological deficit

Right hemisphere deficit syndrome

Scan myocardial perfusion abnormal

Shunt occlusion

Shunt thrombosis

SI QIII TIII pattern

Silent myocardial infarction

Skin ulcer

Soft tissue necrosis

Spinal artery embolism

Spinal artery thrombosis

Spinal cord infarction

Spinal cord ischaemia

Spinal vascular disorder

Splenic artery stenosis

Splenic artery thrombosis

Splenic embolism

Splenic infarction

Splenic thrombosis

Splenic vein occlusion

Splenic vein thrombosis

Spontaneous amputation

Stoma site thrombosis

Stress cardiomyopathy

Stress echocardiogram abnormal

Stroke in evolution

Subclavian artery embolism 
Table 2 (continued)

\section{Preferred term (MEdDRA 21.0)}

Cardiac stress test abnormal

Cardiac ventricular scarring

Cardiac ventricular thrombosis

Cardiopulmonary exercise test abnormal

Cardio-respiratory arrest

Cardiovascular disorder

Cardiovascular insufficiency

Carotid angioplasty

Carotid arterial embolus

Carotid arteriosclerosis

Carotid artery bypass

Carotid artery calcification

Carotid artery disease

Carotid artery insufficiency

Carotid artery occlusion

Carotid artery restenosis

Carotid artery stenosis

Carotid artery stent insertion

Carotid artery stent removal

Carotid artery thrombosis

Carotid endarterectomy

Carotid revascularisation

Catheter site thrombosis

Catheterisation venous

Cavernous sinus thrombosis

Central pain syndrome

Central venous catheterisation

Cerebellar artery occlusion

Cerebellar artery thrombosis

Cerebellar embolism

Cerebellar infarction

Cerebellar ischaemia

Cerebellar stroke

Cerebral arteriosclerosis

Cerebral artery embolism

Cerebral artery occlusion

Cerebral artery restenosis

Cerebral artery stenosis

Cerebral artery thrombosis

Cerebral autosomal dominant arteriopathy with subcortical infarcts and leukoencephalopathy

Cerebral congestion

Cerebral gas embolism

Cerebral hypoperfusion

Cerebral infarction

Cerebral infarction foetal

Cerebral ischaemia

Cerebral microembolism

Cerebral reperfusion injury

Cerebral revascularisation
Ischaemic heart disease prophylaxis

Ischaemic hepatitis

Ischaemic limb pain

Ischaemic mitral regurgitation

Ischaemic nephropathy

Ischaemic neuropathy

Ischaemic pancreatitis

Ischaemic skin ulcer

Ischaemic stroke

Jugular vein occlusion

Jugular vein thrombosis

Kounis syndrome

Lacunar infarction

Lacunar stroke

Lateral medullary syndrome

Leg amputation

Leriche syndrome

Limb amputation

Limb traumatic amputation

Macular ischaemia

Mahler sign

May-Thurner syndrome

Medical device site thrombosis

Mesenteric arterial occlusion

Mesenteric arteriosclerosis

Mesenteric artery embolism

Mesenteric artery stenosis

Mesenteric artery stent insertion

Mesenteric artery thrombosis

Mesenteric phlebosclerosis

Mesenteric vascular insufficiency

Mesenteric vascular occlusion

Mesenteric vein thrombosis

Mesenteric venous occlusion

Microembolism

Microvascular coronary artery disease

Migrainous infarction

Millard-Gubler syndrome

Monoparesis

Monoplegia

Moyamoya disease

Myocardial hypoxia

Myocardial infarction

Myocardial ischaemia

Myocardial necrosis

Myocardial necrosis marker increased

Myocardial reperfusion injury

Myocardial stunning

Necrosis
Subclavian artery occlusion

Subclavian artery stenosis

Subclavian artery thrombosis

Subclavian coronary steal syndrome

Subclavian steal syndrome

Subclavian vein occlusion

Subclavian vein stenosis

Subclavian vein thrombosis

Subendocardial ischaemia

Superior mesenteric artery syndrome

Superior sagittal sinus thrombosis

Superior vena cava occlusion

Superior vena cava syndrome

Surgical vascular shunt

Testicular infarction

Thalamic infarction

Thrombectomy

Thromboangiitis obliterans

Thromboembolectomy

Thrombolysis

Thrombophlebitis

Thrombophlebitis migrans

Thrombophlebitis neonatal

Thrombophlebitis superficial

Thrombosed varicose vein

Thrombosis

Thrombosis corpora cavernosa

Thrombosis in device

Thrombosis mesenteric vessel

Thrombosis prophylaxis

Thrombotic cerebral infarction

Thrombotic microangiopathy

Thrombotic stroke

Thrombotic thrombocytopenic purpura

Thyroid infarction

Toe amputation

Tongue infarction

Transient ischaemic attack

Transverse sinus thrombosis

Troponin I increased

Troponin increased

Troponin T increased

Truncus coeliacus thrombosis

Tumour embolism

Tumour thrombosis

Ultrasonic angiogram abnormal

Ultrasound Doppler abnormal

Umbilical cord occlusion

Umbilical cord thrombosis 
Table 2 (continued)

\section{Preferred term (MEdDRA 21.0)}

Cerebral septic infarct

Cerebral small vessel ischaemic disease

Cerebral thrombosis

Cerebral vascular occlusion

Cerebral vasoconstriction

Cerebral venous thrombosis

Cerebrospinal thrombotic tamponade

Cerebrovascular accident

Cerebrovascular accident prophylaxis

Cerebrovascular disorder

Cerebrovascular insufficiency

Cerebrovascular operation

Cerebrovascular stenosis

Chest discomfort

Chest pain

Choroidal infarction

Choroidal sclerosis

Claudication of jaw muscles

Clumsiness

Coeliac artery occlusion

Coeliac artery stenosis

Colitis ischaemic

Collateral circulation

Compression garment application

Computerised tomogram coronary artery abnormal

Coronary angioplasty

Coronary arterial stent insertion

Coronary artery bypass

Coronary artery compression

Coronary artery disease

Coronary artery dissection

Coronary artery embolism

Coronary artery insufficiency

Coronary artery occlusion

Coronary artery reocclusion

Coronary artery restenosis

Coronary artery stenosis

Coronary artery surgery

Coronary artery thrombosis

Coronary brachytherapy

Coronary bypass stenosis

Coronary bypass thrombosis

Coronary endarterectomy

Coronary no-reflow phenomenon

Coronary ostial stenosis

Coronary revascularisation

Coronary vascular graft occlusion

Coronary vascular graft stenosis

Coronary vein stenosis
Necrosis ischaemic

Nephroangiosclerosis

$\mathrm{NIH}$ stroke scale abnormal

$\mathrm{NIH}$ stroke scale score decreased

$\mathrm{NIH}$ stroke scale score increased

Non-cardiac chest pain

Obstetrical pulmonary embolism

Obstructive shock

Ocular ischaemic syndrome

Ocular vascular disorder

Omental infarction

Ophthalmic vein thrombosis

Optic ischaemic neuropathy

Optic nerve infarction

Ovarian vein thrombosis

Paget-Schroetter syndrome

Pancreatic infarction

Papillary muscle infarction

Paradoxical embolism

Paralysis

Paraneoplastic thrombosis

Paraparesis

Paraplegia

Paresis

Pelvic venous thrombosis

Penetrating atherosclerotic ulcer

Penile artery occlusion

Penile vein thrombosis

Percutaneous coronary intervention

Perinatal stroke

Peripheral arterial occlusive disease

Peripheral arterial reocclusion

Peripheral artery angioplasty

Peripheral artery bypass

Peripheral artery occlusion

Peripheral artery restenosis

Peripheral artery stenosis

Peripheral artery stent insertion

Peripheral artery thrombosis

Peripheral coldness

Peripheral embolism

Peripheral endarterectomy

Peripheral ischaemia

Peripheral revascularisation

Peripheral vascular disorder

Periprocedural myocardial infarction

Phlebectomy

Phlebitis

Phlebosclerosis
Uterine ischaemia

Vaccination site thrombosis

Vascular access site occlusion

Vascular access site thrombosis

Vascular encephalopathy

Vascular graft

Vascular graft occlusion

Vascular graft restenosis

Vascular graft stenosis

Vascular graft thrombosis

Vascular insufficiency

Vascular occlusion

Vascular operation

Vascular pseudoaneurysm thrombosis

Vascular shunt

Vascular skin disorder

Vascular stenosis

Vascular stent insertion

Vascular stent occlusion

Vascular stent restenosis

Vascular stent stenosis

Vascular stent thrombosis

Vasculitis

Vasoconstriction

Vasodilation procedure

Vena cava embolism

Vena cava filter insertion

Vena cava filter removal

Vena cava thrombosis

Venogram abnormal

Venoocclusive disease

Venoocclusive liver disease

Venous angioplasty

Venous occlusion

Venous operation

Venous recanalisation

Venous repair

Venous stenosis

Venous stent insertion

Venous thrombosis

Venous thrombosis in pregnancy

Venous thrombosis limb

Venous thrombosis neonatal

Vertebral artery occlusion

Vertebral artery stenosis

Vertebral artery thrombosis

Vertebrobasilar insufficiency

Vessel puncture site occlusion

Vessel puncture site thrombosis 
Table 2 (continued)

\section{Preferred term (MEdDRA 21.0)}

Deep vein thrombosis

Deep vein thrombosis postoperative

Delayed ischaemic neurological deficit

Dependent rubor

Device embolisation

Device occlusion

Device related thrombosis

Diabetic macroangiopathy

Diabetic microangiopathy

Diabetic vascular disorder
Vestibular ischaemia

Visceral venous thrombosis

Visual acuity reduced transiently

Visual agnosia

Visual midline shift syndrome

Wall motion score index abnormal
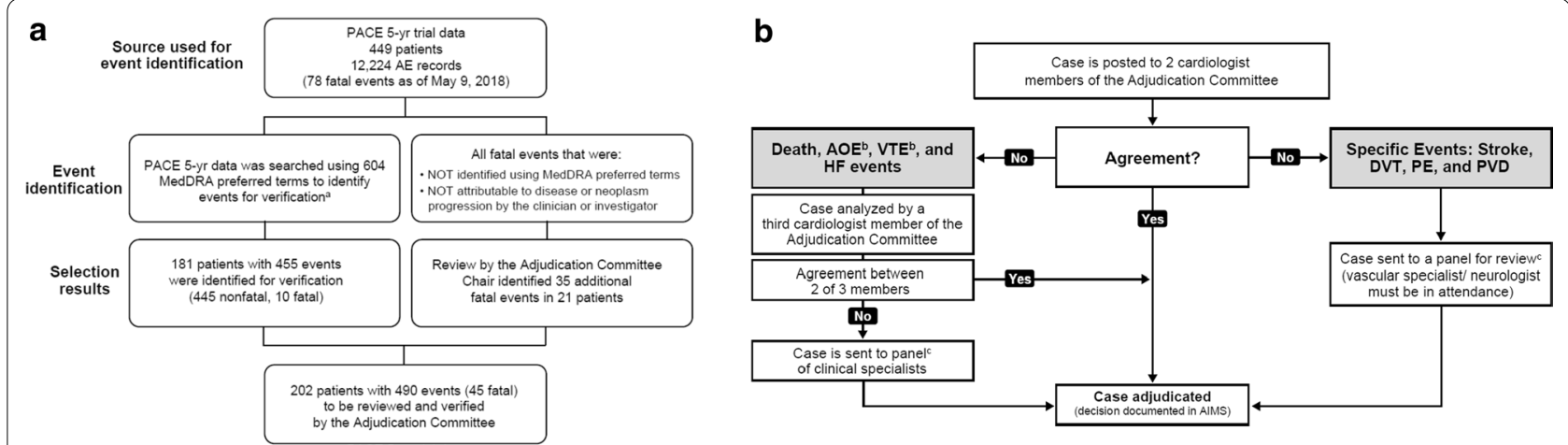

Fig. 1 CONSORT flow diagram and process for adjudication of arterial occlusive events (AOEs). A CONSORT diagram: Identification of AOEs for review by the adjudication committee. B Adjudication process flow charts. AE adverse event, AC adjudication committee, AIM Applied Clinical Intelligence Information Management System, MedDRA Medical Dictionary for Regulatory Activities, PACE Ponatinib Ph+ ALL and CML Evaluation,

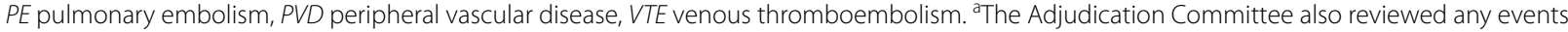
included in the Cardiac Failure Standard MedDRA Query (SMQ) to determine whether any heart failure events were AOEs. ${ }^{b}$ AOEs evaluated on the left panel excluded events evaluated in the right panel (stroke, DVT, and PE). ${ }^{C}$ Per the charter, panel meetings were convened to discuss events for which a decision was not reached via independent voting. The quorum for panel meeting attendance was dependent on the type of event(s) to be discussed (i.e., cardiologist, neurologist, or vascular specialist)

"non-cardiac chest pain" or "claudication") with a low severity level and no accompanying changes in medication or hospitalization were adjudicated to not be AOEs unless they had an anatomic diagnosis provided (e.g., "severe superficial femoral artery stenosis"). If the term "infarction" was provided for stroke events, the adjudicators categorized the event as ischemic stroke. Revascularization was not always clearly reported by investigators.

\section{Statistics}

Exposure-adjusted AOE rates were calculated as: (number of first events in interval)/(total exposure for interval in patient-years) $\times 100$. The relative risk of serious AOEs was analyzed by baseline risk category in patients from the safety population for whom all baseline risk categories were available. Risk categories included commonly recognized cardiovascular risk factors for which data were collected (arterial hypertension, hypercholesterolemia, diabetes mellitus, and obesity), and history of heart disease (non-ischemic or ischemic).

\section{Results}

\section{Patient disposition and baseline characteristics}

Patient disposition and baseline characteristics in the PACE trial have been published [2, 3]. A total of 449 patients, including $270 \mathrm{CP}-\mathrm{CML}$ patients, 85 accelerated-phase (AP) CML patients, 62 blast-phase (BP) CML patients, and $32 \mathrm{Ph}+$ ALL patients, were enrolled 
Table 3 Adjudication committee prespecified definitions of events

Events

Cardiovascular (CV) death

Death associated with acute myocardial infarction

Sudden cardiac death

Death due to HF

Death due to stroke

Death due to CV procedures

Death due to CV hemorrhage

Death due to other CV causes

Non-CV death

\section{Definitions}

The cause of death will be determined by the principal condition that caused the death, not the immediate mode of death. Members of the adjudication committee will review all available information and use their clinical expertise to adjudicate the cause of death

$\mathrm{CV}$ death includes death resulting from an acute myocardial infarction (MI), sudden cardiac death, death due to heart failure (HF), death due to stroke, death due to CV procedures, death due to CV hemorrhage, death due to pulmonary embolism, and death due to other CV causes

Refers to a death by any CV mechanism (e.g., arrhythmia, sudden death, heart failure, stroke, pulmonary embolus, peripheral arterial disease) $\leq 30$ days after a Ml related to the immediate consequences of the $\mathrm{Ml}$, such as progressive heart failure or recalcitrant arrhythmia. Acute Ml should be verified to the extent possible by the diagnostic criteria outlined for acute MI (see below) or by autopsy findings showing recent Ml or recent coronary thrombosis

Death resulting from a procedure to treat a $\mathrm{MI}$ (percutaneous coronary intervention ( $\mathrm{PCI}$ ), coronary artery bypass graft surgery (CABG), or to treat a complication resulting from $\mathrm{Ml}$, should also be considered death due to acute $\mathrm{Ml}$

Death resulting from an elective coronary procedure to treat myocardial ischemia (i.e., chronic stable angina) or death due to a $\mathrm{Ml}$ that occurs as a direct consequence of a CV investigation/procedure/operation should be considered as a death due to a CV procedure

Sudden cardiac death refers to death that occurs unexpectedly, not following an acute MI (as defined above) and includes the following deaths:

Witnessed and occurring without new or worsening symptoms

Witnessed within 60 min of the onset of new or worsening cardiac symptoms, unless the symptoms suggest acute Ml

Witnessed and attributed to an identified arrhythmia (e.g., captured on an electrocardiographic (ECG) recording or witnessed on a monitor, or unwitnessed but found on implantable cardioverter-defibrillator review)

After unsuccessful resuscitation from cardiac arrest (e.g., implantable cardioverter-defibrillator [ICD] unresponsive sudden cardiac death, pulseless electrical activity arrest)

After successful resuscitation from cardiac arrest and without identification of a specific cardiac or non-cardiac etiology

Unwitnessed death in a subject seen alive and clinically stable $\leq 24 \mathrm{~h}$ prior to being found dead without any evidence supporting a specific non-CV cause of death (information regarding the patient's clinical status preceding death should be provided, if available)

Note: Unless additional information suggests an alternate specific cause of death (e.g., Death due to other CV causes), if a patient is seen alive $\leq 24 \mathrm{~h}$ of being found dead, sudden cardiac death should be recorded. For patients who were not observed alive within $24 \mathrm{~h}$ of death, undetermined cause of death should be recorded (e.g., a subject found dead in bed, but who had not been seen by family for several days)

Note: Successful resuscitation without death should be captured as a resuscitated sudden cardiac death in the non-fatal voting flow

Refers to death associated with clinically worsening symptoms and/or signs of HF regardless of etiology. Deaths due to HF can have various etiologies, including single or recurrent Mls, ischemic or non-ischemic cardiomyopathy, hypertension, or valvular disease

Note: Due to the pro-thrombotic nature of the subject population, a thrombo-embolic option is included during voting. See rules in the non-fatal heart failure definition

Refers to death within 30 days that is either a direct consequence of the stroke or a complication of the stroke. Acute stroke should be verified to the extent possible by the diagnostic criteria outlined for stroke

Refers to death caused by the immediate complications of a cardiac procedure not in the context of treatment for acute $\mathrm{Ml}$

Refers to death related to hemorrhage such as a non-stroke intracranial hemorrhage, non-procedural or nontraumatic vascular rupture (e.g., aortic aneurysm), or hemorrhage causing cardiac tamponade

Refers to a CV death not included in the above categories but with a specific, known cause (e.g., pulmonary embolism or peripheral vascular disease (venous or arterial disease)

Non-CV death is defined as any death with a specific cause that is not thought to be of CV nature. Adjudication committee members will be asked to indicate the most likely cause of non-cardiovascular death on their voting form 
Table 3 (continued)

Events Definitions

Events

Examples of non-CV death are: pulmonary causes, renal causes, gastrointestinal causes, hepatobiliary causes, pancreatic causes, infection (including sepsis), inflammatory (e.g., systemic inflammatory response syndrome $(\mathrm{SIRS})$ )/immune (including autoimmune)(may include anaphylaxis from environmental (e.g., food allergies), hemorrhage that is neither cardiovascular bleeding or stroke, non-CV procedure or surgery, trauma, suicide, non-prescription drug reaction or overdose, prescription drug reaction or overdose (many include anaphylaxis), neurological (non-cardiovascular), malignancy (i.e., new malignancy, worsening of prior malignancy) or other (should be specified)

Undetermined cause of death

Undetermined cause of death refers to a death not attributable to one of the above categories. Inability to classify the cause of death may be due to lack of information (e.g., the only available information is "patient died") or when there is insufficient supporting information or detail to assign the cause of death. In general, most deaths should be classifiable as CV or non-CV, and the use of this category of death, therefore, should be discouraged and should apply to few patients in well-run clinical trials

Non-fatal event definitions Myocardial infarction (non-fatal)

Criteria for acute MI: The term MI should be used when there is evidence of myocardial necrosis in a clinical setting consistent with acute myocardial ischemia. In general Ml is defined as a combination of evidence of myocardial necrosis (changes in cardiac biomarkers) and supporting information (derived from the clinical presentation, electrocardiographic changes or the results of a myocardial or coronary artery imaging). Under these conditions, any one of the following criteria A to $\mathrm{G}$ meets the diagnosis for MI

Spontaneous MI (type 1): To identify a type $1 \mathrm{Ml}$, patients should demonstrate spontaneous symptoms of myocardial ischemia unprovoked by supply/demand inequity, together with at least one of the following criteria:

Cardiac biomarker elevation: Troponin is the preferred marker for use to adjudicate the presence of acute MI. At least one value should show a rise and/or fall above the lowest cut-point providing $10 \%$ imprecision (typically the upper reference limit for the troponin run per standard of clinical care). Creatine kinase-MB is a secondary choice to troponin; a rise of CK-MB above the local upper reference limit would be consistent with myocardial injury. Total CK may be used in the absence of CK-MB and troponin

Imaging evidence of new non-viable myocardium or new wall motion abnormality

ECG changes consistent with new ischemic changes

ECG changes indicative of new ischemia [new ST-T changes or new left bundle branch block (LBBB)]*

Development of pathological Q-waves in the ECG**

*ECG manifestations of acute myocardial ischemia (in absence of left ventricular hypertrophy (LVH) and left bundle branch block (LBBB)):

ST elevation: New ST elevation at the J-point in two contiguous leads with the cut-off points: $\geq 0.2 \mathrm{mV}$ in men or $\geq 0.15 \mathrm{mV}$ in women in leads V2-V3 and/or $\geq 0.1 \mathrm{mV}$ in other leads

ST depression and T-wave changes: New horizontal or down- sloping ST depression $\geq 0.05 \mathrm{mV}$ in two contiguous leads; and/or T inversion $\geq 0.1 \mathrm{mV}$ in two contiguous leads with prominent R-wave or R/S ratio > 1

**Pathological Q-waves:

Any Q-wave in leads V2-V3 $\geq 0.02$ s or QS complex in leads V2 and V3

Q-wave $\geq 0.03 \mathrm{~s}$ and $\geq 0.1 \mathrm{mV}$ deep or QS complex in leads I, II, aVL, aVF, or V4-V6 in any two leads of a contiguous lead grouping (I, aVL, V6; V4-V6; II, III, and aVF)

"Demand" related MI (type 2): Patients with type 2 MI should be considered with similar diagnostic criteria as a type $1 \mathrm{Ml}$, however type $2 \mathrm{Ml}$ should be considered present when myocardial ischemia and infarction are consequent to supply/demand inequity, rather than a spontaneous plaque rupture and coronary thrombosis Percutaneous coronary intervention-related MI (type 4a): For percutaneous coronary interventions (PCI) in patients with normal baseline troponin values, elevations of cardiac biomarkers above the 99th percentile URL, within $24 \mathrm{~h}$ of the procedure, are indicative of peri-procedural myocardial necrosis. By convention, increases of biomarkers greater than $5 \times 99$ th percentile URL (Troponin or CK-MB $>5 \times 99$ th percentile URL) are consistent with PCl-related MI. If the cardiac biomarker is elevated prior to $\mathrm{PCl}, \mathrm{a} \geq 20 \%$ increase of the value in the second cardiac biomarker sample within $24 \mathrm{~h}$ of the $\mathrm{PCl}$ and documentation that cardiac biomarker values were decreasing ( 2 samples at least $6 \mathrm{~h}$ apart) prior to the suspected recurrent $\mathrm{Ml}$ is also consistent with $\mathrm{PCl}$-related MI. In addition to biomarker elevation one of the following must exist:

Symptoms suggestive of myocardial ischemia

New ischemic ECG changes or new LBBB

Angiographic findings consistent with procedural complication (e.g., Loss of patency, persistent slow/nonflow or embolization)

Imaging demonstration of new loss of viable myocardium or new regional wall motion abnormality

Ml associated with stent thrombosis or stent restenosis as documented by angiography or at autopsy will also be captured as subtypes $4 \mathrm{~b}$ and $4 \mathrm{c}$ 
Table 3 (continued)

\section{Events}

\section{Definitions}

Stent thrombosis related MI (type 4b): Ml associated with stent thrombosis as detected by coronary angiography or at autopsy, where symptoms suggestive of myocardial ischemia are present, and with a rise and/or fall of cardiac biomarker values with at least 1 value > 99th percentile of the URL. If found with autopsy, it will be captured under cardiac death

Definite stent thrombosis is considered to have occurred by either angiographic or pathological confirmation:

Angiographic confirmation of stent thrombosis (Incidental angiographic documentation of stent occlusion in the absence of clinical signs or symptoms is not considered a confirmed stent thrombosis [silent occlusion]). The presence of a thrombus (intracoronary) that originates in the stent or in the segment $5 \mathrm{~mm}$ proximal or distal to the stent and presence of at least 1 of the following criteria within a 48-h time window:

Acute onset of ischemic symptoms at rest

New ischemic ECG changes that suggest acute ischemia

Typical rise and fall in cardiac biomarkers (refer to definition of spontaneous MI)

Non-occlusive thrombus

Intracoronary thrombus is defined as a (spheric, ovoid, or irregular) non-calcified filling defect or lucency surrounded by contrast material (on 3 sides or within a coronary stenosis) seen in multiple projections, or persistence of contrast material within the lumen, or a visible embolization of intraluminal material downstream

Occlusive thrombus TIMI 0 or TIMI 1 intrastent or proximal to a stent up to the most adjacent proximal side branch or main branch (if originates from the side branch)

Pathological confirmation of stent thrombosis: Evidence of recent thrombus within the stent determined at autopsy or via examination of tissue retrieved following thrombectomy

Probable stent thrombosis: Clinical definition of probable stent thrombosis is considered to have occurred after intracoronary stenting in the following cases:

Any unexplained death within the first 30 days

Irrespective of the time after the index procedure, any Ml that is related to documented acute ischemia in the territory of the implanted stent without angiographic confirmation of stent thrombosis and in the absence of any other obvious cause

Stent restenosis-related $\mathrm{MI}$ (type 4c): Ml associated with stent restenosis as detected by coronary angiography or at autopsy, occurring $>48 \mathrm{~h}$ after index $\mathrm{PCl}$ without evidence of stent thrombosis but with symptoms suggestive of myocardial ischemia, and with elevation of cardiac biomarker values to > 99th percentile of the URL. This classification also requires the following:

Does not meet criteria for any other classification of $\mathrm{Ml}$

Presence of $\mathrm{a} \geq 50 \%$ stenosis at the site of previous successful stent $\mathrm{PCl}$ or a complex lesion and no other significant obstructive CAD of greater severity following:

Initially successful stent deployment

$\mathrm{OR}$

Dilatation of a coronary artery stenosis with balloon angioplasty to $<50 \%$ stenosis

If found with autopsy, it will be captured under cardiac death

Coronary artery bypass grafting-related MI (type 5): MI associated with CABG is arbitrarily defined by elevation of cardiac biomarker values $>10 \times 99$ th percentile URL in patients with normal baseline cardiac biomarker values ( $\leq 99$ th percentile URL). In addition to any one of the following:

New pathological Q-waves or new LBBB

Angiographic documented new graft or new native coronary artery occlusion

Imaging evidence of new loss of viable myocardium or new regional wall motion abnormality

Heart failure event

A heart failure event includes hospitalization for heart failure and may include any urgent outpatient visits for heart failure. The date of this event will be the day of hospitalization of the patient (including any overnight stay at the emergency room or chest pain unit) or the day of visit to the urgent outpatient center. Due to the prothrombotic nature of the subject population, a thrombo-embolic option is included during voting

The following rules may be applied to indicate if heart failure is attributed to an AOENTE:

Heart failure may be attributed to an AOENTE if related to coronary artery disease, hypertension, cardiomyopathy or myocardial infarction

The relationship of heart failure to an AOENTE may be excluded if the underlying cause of heart failure is heart valve disorders, congenital heart disorders or arrhythmias

Heart failure requiring hospitalization Heart failure hospitalization is defined as an event that meets all the following criteria:

Patient is admitted to the hospital with a primary diagnosis of HF 
Table 3 (continued)

\section{Definitions}

Patient's length of stay in hospital extends for at least $24 \mathrm{~h}$ (or a change in calendar date if the hospital admission and discharge times are unavailable)

Patient exhibits documented new or worsening symptoms due to HF on presentation, including at least ONE of the following:

Dyspnea

Dyspnea with exertion

Orthopnea

Paroxysmal nocturnal dyspnea

Decrease exercise tolerance

Fatigue

Other symptoms of worsened end-organ perfusion or volume overload

Patient has objective evidence of new/worsening HF, consisting of at least TWO physical examination findings OR one physical examination finding and at least one laboratory criterion, including:

Physical examination findings considered to be due to heart failure

Peripheral edema

Increasing abdominal distention or ascites (in the absence of primary hepatic disease)

Pulmonary rales/crackles/crepitations

Increased jugular venous pressure and/or hepatojugular reflux

$S^{3}$ gallop

Clinically significant or rapid weight gain thought to be related to fluid retention

Laboratory evidence of new or worsening HF, if obtained within $24 \mathrm{~h}$ of presentation, including:

Increased b-type natriuretic peptide (BNP)/N-terminal proBNP (NT-proBNP) concentrations consistent with decompensation of heart failure (such as BNP $>500 \mathrm{pg} / \mathrm{mL}$ or NT-proBNP $>1800 \mathrm{pg} / \mathrm{mL}$ ). In patients with chronically elevated natriuretic peptides, a significant increase should be noted above baseline

Radiological evidence of pulmonary congestion

New or worsened bilateral pleural effusions

Noninvasive diagnostic evidence of clinically significant elevated left or right-sided ventricular filling pressure or low cardiac input

Invasive diagnostic evidence with right heart catheterization showing a pulmonary capillary wedge pressure (pulmonary artery occlusion pressure) $\geq 18 \mathrm{mmHg}$, central venous pressure $\geq 12 \mathrm{mmHg}$, or a cardiac index $<2.2 \mathrm{~L} / \mathrm{min} / \mathrm{m}^{2}$

Patient receives initiation or intensification of treatment specifically for HF (at least one of the following):

Augmentation in oral diuretic therapy or ACE inhibitor

Intravenous diuretic or vasoactive agent (e.g., inotrope, vasopressor, or vasodilator)

Mechanical or surgical intervention:

Mechanical circulatory support (e.g., intra-aortic balloon pump, ventricular assist device, extracorporeal membrane oxygenation, total artificial heart)

Mechanical fluid removal (e.g., dialysis, ultrafiltration, hemofiltration)

Urgent heart failure visit

Hospitalization for unstable angina
An urgent heart failure visit is defined as an event that meets all the following criteria:

The patient has an urgent, unscheduled office/practice or emergency department visit for a primary diagnosis of heart failure, but not meeting the criteria for a heart failure hospitalization

All signs/symptoms for heart failure hospitalization (i.e., symptoms, physical examination findings/lab evidence of new or worsening HF as indicated under definition for Heart Failure Hospitalization) must be met The patient receives initiation or intensification of treatment specifically for heart failure, as detailed in the heart failure hospitalization section with the exception of oral diuretic therapy (which will not be sufficient)

The date of this event will be the day of hospitalization of the patient including any overnight stay at an emergency room or chest pain unit

Hospitalization for unstable angina is defined as an event that meets all the following criteria:

Negative cardiac biomarkers and no evidence of acute MI

Ischemic discomfort (angina or other symptoms thought to be equivalent) $\geq 10 \mathrm{~min}$ in duration occurring at rest or in an accelerating pattern with frequent episodes associated with progressively decreased exercise capacity 
Table 3 (continued)

Events

Stroke

\section{Definitions}

Unscheduled hospitalization within $24 \mathrm{~h}$ of the most recent symptoms. Hospitalization is defined as an admission to an inpatient unit or a visit to an emergency department that results in at least a $24 \mathrm{~h}$ stay (or a change in calendar date if the hospital admission or discharge times are not available)

At least one of the following:

New or worsening ST or T-wave changes on resting ECG (in absence of confounders such as LBBB or LVH)

ST Elevation: New transient (duration $<20 \mathrm{~min}$ ) at the J point in two contiguous leads with the cutpoints: $\geq 0.1 \mathrm{mV}$ in all leads other than leads V2-V3 where the following cut-points apply: $\geq 0.2 \mathrm{mV}$ in men $\geq 40$ years ( $\geq 0.25 \mathrm{mV}$ in men $<40$ years) or $\geq 0.15 \mathrm{mV}$ in women

ST depression and T-wave changes: New horizontal or down-sloping ST depression $\geq 0.05 \mathrm{mV}$ in two contiguous leads and/or a new T inversion $\geq 0.3 \mathrm{mV}$ in two contiguous leads with prominent $\mathrm{R}$-wave or R/S ratio $>1$

Definite evidence of inducible myocardial ischemia as demonstrated by one of the following and believed to be responsible for symptoms:

Early positive stress test (defined as ST elevation or $\geq 2 \mathrm{~mm}$ ST depression prior to 5 mets)

Stress echocardiography (reversible wall motion abnormality)

Myocardial scintigraphy (reversible perfusion defect)

MRI (myocardial perfusion deficit under pharmacologic stress)

Angiographic evidence of new or worse $\geq 70 \%$ lesion ( $\geq 50 \%$ for left main lesion) and/or thrombus in an epicardial coronary artery that is believed to be responsible for the myocardial ischemic symptoms/signs

Need for coronary revascularization procedure ( $\mathrm{PCl}$ or $\mathrm{CABG}$ ) for the presumed culprit lesion(s). This criterion would be fulfilled if revascularization was undertaken during the unscheduled hospitalization, or subsequent to transfer to another institution without interceding home discharge

Stroke is defined as an acute episode of focal or global neurological dysfunction caused by brain, spinal cord, or retinal vascular injury as a result of hemorrhage or infarction. Strokes will be classified as ischemic, hemorrhagic, retinal artery occlusion or thrombosis or undetermined

General

Stroke is defined as an acute episode of focal or global neurological dysfunction caused by brain, spinal cord, or retinal vascular injury as a result of hemorrhage or infarction, with symptom duration of $24 \mathrm{~h}$ or more. Episodes lasting less than $24 \mathrm{~h}$ can be considered a stroke if there is an intervention to abort the stroke (e.g., thrombolytic therapy), diagnostic confirmation of the stroke, or patient death prior to reaching the $24 \mathrm{~h}$ duration

Subdural and epidural hematomas are intracranial hemorrhagic events and are not strokes

Diagnosis of stroke

For the diagnosis of stroke, the following 4 criteria should be fulfilled:

Acute onset* of a focal/global neurological deficit with at least one of the following:

Change in level of consciousness

Hemiplegia

Hemiparesis

Numbness or sensory loss affecting one side of the body

Dysphasia/Aphasia

Hemianopia (loss of half of the field of vision of one or both eyes)

Other new neurological sign(s)/symptom(s) consistent with stroke

* If the mode of onset is uncertain, a diagnosis of stroke may be made provided that there is no plausible nonstroke cause for the clinical presentation

Duration of a focal/global neurological deficit $\geq 24 \mathrm{~h}$

$\mathrm{OR}$

\section{$<24 \mathrm{~h}$ if}

This is because of at least one of the following therapeutic interventions:

Pharmacologic (i.e., thrombolytic drug administration)

Non-pharmacologic (i.e., neurointerventional procedure (e.g., intracranial angioplasty))

or

available brain imaging clearly documents a new hemorrhage or infarct

or

the neurological deficit results in death 
Table 3 (continued)

\section{Definitions}

No other readily identifiable non-stroke cause for the clinical presentation (e.g., brain tumor, trauma, infection, hypoglycemia, peripheral lesion)

Confirmation of the diagnosis by at least one of the following:**

Neurology or neurosurgical specialist

Brain imaging procedure (at least one of the following):

CTscan

MRI scan

Cerebral vessel angiography

Lumbar puncture (i.e., spinal fluid analysis diagnostic of subarachnoid hemorrhage)

**If a stroke is reported but evidence of confirmation of the diagnosis by the methods outlined above is absent, the event will be discussed at a full EAC meeting. In such cases, the event may be adjudicated as a stroke on the basis of the clinical presentation alone, but full EAC consensus will be mandatory

Classification of stroke

Strokes are sub-classified as follows:

Ischemic (non-hemorrhagic)

Ischemic stroke is defined as an acute episode of focal cerebral, spinal or retinal dysfunction caused by infarction of central nervous system tissue. Hemorrhage may be a consequence of ischemic stroke. In this situation the stroke is an ischemic stroke with hemorrhagic transformation and not a hemorrhagic stroke

Hemorrhagic

Hemorrhagic stroke is defined as an acute episode of focal or global cerebral or spinal dysfunction caused by intraparenchymal, intraventricular, or subarachnoid hemorrhage

Retinal artery occlusion or thrombosis

Retinal artery occlusion or thrombosis is defined as a blockage in one of the retinal arteries

Occlusions may be caused by a thromboembolism or other risk factors such as atherosclerosis and arrhythmias

Note: Amaurosis fugax is not considered part of this endpoint

Undetermined stroke

Undetermined stroke is defined as an acute episode of focal or global neurological dysfunction caused by presumed brain, spinal cord, as a result of hemorrhage or infarction but with insufficient information to allow categorization as \#1 and \#2 above

Note: Given the scope of this study, stroke disability will not be measured. TIA definition was intentionally left out for this study; suspected TIA events will be identified for adjudication in order to rule out stroke

Venous thrombosis

Deep vein thrombosis
Superficial vein thrombosis

Superficial vein thrombosis (SVT) refers to a blood clot in one of the superficial veins near the surface of the body. There is usually an inflammatory reaction around the vein and may present with as a painful induration with erythema. An SVT can lead to a serious complication such as a higher risk for pulmonary embolism

Superficial vein thrombosis could be documented by one of the following:

Clinical symptoms (such as warmth, edema, 'cord-like' palpable mass, erythema, pain)

Duplex ultrasound

Deep vein thrombosis (DVT) refers to a blood clot in one of the deep veins (to include distal and proximal DVT). It may occur anywhere in the body but is most common in the extremities, a clot blocks blood circulation through these veins, which carry blood back to the heart. This commonly causes pain and swelling distal to the thrombus. Severe complications of DVT may occur when a clot embolizes to the lung

Deep vein thrombosis could be documented by one of the following:

Venous ultrasonography

Compression ultrasonography (CUS)

Impedance plethysmography (IPG)

Venography

CT scan

MRI

At autopsy

Location

Venous thrombosis (DVT and SVT) will be categorized for location by the EAC 
Table 3 (continued)

\begin{tabular}{ll}
\hline Events & Definitions \\
\hline Members as follows: \\
Lower limb \\
Upper limb \\
Retinal vein \\
Abdominal viscera \\
Other (e.g., more unusual sites of cerebral venous thrombosis)
\end{tabular}

Pulmonary embolism

Other AOENTE

Revascularization procedures
A pulmonary embolism (PE) is a blood clot in the arteries of the lung that typically arise from the veins. The embolus not only prevents the exchange of oxygen and carbon dioxide via the lungs, but it also decreases blood supply to the lung tissue itself, potentially causing infarction. The most common symptoms include pleuritic chest pain, dyspnea, and hemoptysis. A PE may lead to sudden death. Death due to PE refers to death that is either a direct consequence or complication of a PE. Fatal PE is captured in the fatal definition section as death due to other CV causes

Pulmonary embolism should be documented by supporting evidence found within any one of the following:

CT scan

Pulmonary angiogram

Ventilation/perfusion lung scan (VPLS)

Inconclusive spiral CT, pulmonary angiography or lung scintigraphy with demonstration of DVT in the lower extremities by CUS or venography with clinical, lab and EKG findings consistent with PE

At autopsy

Peripheral vascular disease (PVD)

Peripheral vascular disease refers to a blood circulation disorder outside of the heart and brain that causes the blood vessels to block, narrow or spasm. PVD can be either in veins or arteries. Physical symptoms may include weak pulses, wounds/ulcers that won't heal, thin or pale skin

PVD could be documented by one of the following:

Doppler ultrasound

Ankle-brachial index

Angiography

Magnetic resonance angiography

Computerized tomography angiography

Members will be asked to choose if this is a venous or arterial occlusive event

For fatal and non-fatal cardiovascular endpoint events, members must also indicate if the event is associated with a revascularization procedure ( $\mathrm{PCl}, \mathrm{CABG}$ or $\mathrm{PVI})$

Percutaneous coronary intervention (PCI)

Defined as the placement of an angioplasty guidewire, balloon, or other device (e.g., stent, atherectomy, brachytherapy or thrombectomy catheter) into a native coronary artery or CABG for the purpose of mechanical coronary revascularization. The assessment of coronary lesion severity by intravascular ultrasonography, coronary flow reserve, or fractional flow reserve is not considered a $\mathrm{PCl}$ procedure

Coronary artery bypass graft (CABG)

Defined as a procedure performed to bypass partially or completely occluded coronary arteries with veins and/ or arteries harvested from elsewhere in the body, thereby improving the blood supply to the coronary circulation supplying the myocardium

Peripheral vascular intervention (PVI)

Peripheral vascular intervention is a catheter-based or open surgical procedure designed to improve arterial or venous blood flow or otherwise modify or revise vascular conduits. Procedures may include, but are not limited to percutaneous transluminal balloon angioplasty, stent placement, thrombectomy, embolectomy, atherectomy, dissection repair, aneurysm exclusion, treatment of dialysis conduits, placement of various devices, intravascular thrombolysis or other pharmacotherapies, and open surgical bypass or revision 
between September 2010 and October 2011. Baseline characteristics are summarized in Table 4. Among all 449 patients, the median age was 59 years and $53 \%$ of patients were male. Most (93\%) patients had received 2 or more prior TKIs. At baseline, $53 \%$ of patients had arterial hypertension, $49 \%$ had hypercholesterolemia, and $24 \%$ had $B M I \geq 30 \mathrm{~kg} / \mathrm{m}^{2}$. Forty-three percent of patients had a baseline history of non-ischemic cardiac disease, and $23 \%$ had a history of ischemic cardiovascular disease. Safety data reviewed by the adjudication committee reflect data collected as of February 6, 2017, with median follow-up of 37.3 months for all patients and 56.8 months (range 0.1-73.1 months) for CP-CML patients.

\section{Adjudication results}

Rates of adjudicated AOEs were lower than rates of non-adjudicated AOEs (Fig. 2A). Overall, 17\% (78/449) of patients had adjudicated AOEs compared with 25\% $(111 / 449)$ with non-adjudicated AOEs. Most patients with serious AOEs were adjudicated as having serious AOEs (20\% [90/449] non-adjudicated vs. 16\% [74/449] adjudicated). Most (95\% [74/78]) patients with adjudicated AOEs had serious AOEs. In CP-CML patients, rates of adjudicated AOEs (21\% [57/270]) were also lower than rates of non-adjudicated AOEs (31\% [84/270]); 95\% [54/57] of CP-CML patients with adjudicated AOEs had serious AOEs. The rates of AOEs by AOE type (i.e., cardiovascular, cerebrovascular, and peripheral vascular) are presented for all patients in Table 5 and for CP-CML patients in Table 6.

The most common non-adjudicated and adjudicated AOEs and serious AOEs are summarized in Table 7. The most common $(>2 \%)$ non-adjudicated AOEs were angina pectoris $(6 \% ; 28 / 449)$, peripheral arterial occlusive disease $(5 \% ; 22 / 449)$, MI $(4 \% ; 18 / 449)$, coronary artery disease (3\% [14/449]). The only adjudicated AOE reported in $>2 \%$ of patients was peripheral arterial occlusive disease (4\% [16/449]). Non-adjudicated AOEs that were most commonly adjudicated as not AOEs were angina pectoris, non-cardiac chest pain, and chest pain, as these events were often recorded as symptoms (e.g., "non-cardiac chest pain" or "claudication") or presumptive diagnoses with a low severity level and no accompanying changes in medication or hospitalization.

The exposure-adjusted incidence of adjudicated AOEs (8.9 patients with events per 100 patient-years) and serious AOEs (8.4 patients with events per 100 patientyears) was lower than the exposure-adjusted incidence of non-adjudicated AOEs (11.3 and 9.2 per 100 patientyears, respectively). The exposure-adjusted incidence of newly occurring AOEs decreased over time (Fig. 2B). The median time to onset of the first adjudicated AOE was 14.1 months (range: 0.1 to 49.5 ; Table 8 ).

\section{Resolution of AOEs, dose modifications, and discontinuations}

Among the 78 patients with an adjudicated AOE, events resolved in 51 patients. Among 43 patients with just one AOE, 74\% (32/43) had resolution of the event; 35 patients had multiple AOEs recorded, with 54\% (19/35) patients having resolution of all the events. Most patients continued ponatinib after the AOE, including 36 patients (46\%) who continued ponatinib without dose modification and 27 patients (35\%) who had their doses reduced and/or interrupted after the event (Table 9). Seven patients (9\%) discontinued ponatinib due to an adjudicated AOE. Rates of dose modifications following AOEs are summarized in Table 9.

\section{Risk factor analysis}

The most common baseline risk factors in patients who developed an AOE were arterial hypertension and hypercholesterolemia (Table 10). Patients with adjudicated AOEs also had higher rates of concomitant use of antihypertensive medications, platelet aggregation inhibitor medications, and anti-diabetic agents compared with patients who did not have AOEs (Table 11).

The incidence of adjudicated AOEs by number of baseline risk factors (including arterial hypertension, hypercholesterolemia, obesity, diabetes mellitus, non-ischemic cardiac disease, and ischemic disease) is shown in Fig. 2C. The rate of adjudicated AOEs was 13\% (24/189) among patients with 1-2 risk factors, and 29\% (52/180) among patients with 3 or more risk factors. Of the 80 patients without any risk factors at baseline, only $2(3 \%)$ had an AOE.

\section{Fatal AOEs}

Separate adjudication of deaths revealed that 11 adjudicated AOEs were associated with death. These included 2 cases of cardiac arrest and 1 each of the following: bradycardic arrest, cardiac failure, intracranial hemorrhage, worsening of congestive heart failure, superior mesenteric artery occlusion, hemorrhagic cerebral infarction, congestive heart failure, ischemic stroke, and acute anterior myocardial infarction. Nine of the 11 patients with AOEs associated with death had a history of cardiovascular events and/or cardiovascular risk factors recorded at baseline (Table 12). The long-term survival of patients 
Table 4 Baseline characteristics and disposition at end-of-study ${ }^{3}$

\begin{tabular}{|c|c|c|}
\hline & $\begin{array}{l}\text { CP-CML } \\
n=270\end{array}$ & $\begin{array}{l}\text { Total } \\
N=449\end{array}$ \\
\hline \multicolumn{3}{|l|}{ Characteristic at baseline } \\
\hline Median age (range), y & $60(18-94)$ & $59(18-94)$ \\
\hline Female, $n(\%)$ & $126(47)$ & $211(47)$ \\
\hline \multicolumn{3}{|l|}{ Previous use of approved TKIs, $n(\%)^{\mathrm{a}}$} \\
\hline$\geq 2$ drugs & $251(93)$ & $417(93)$ \\
\hline$\geq 3$ drugs & $154(57)$ & $250(56)$ \\
\hline Median duration of previous treatment with approved TKIs (range), $\mathrm{y}^{\mathrm{a}}$ & $5.4(0.4-13.3)$ & $4.6(0.1-13.3)$ \\
\hline \multicolumn{3}{|l|}{ Resistant or intolerant to dasatinib or nilotinib, $n(\%)$} \\
\hline Resistant & $215(80)$ & $375(84)$ \\
\hline Intolerant only & $39(14)$ & $49(11)$ \\
\hline Both resistant and intolerant & $52(19)$ & $81(18)$ \\
\hline \multicolumn{3}{|l|}{ Mutation status, $n(\%)^{b}$} \\
\hline No mutation detected & $138(51)$ & $198(44)$ \\
\hline BCR::ABL1 $1^{T 3151}$ & $64(24)$ & $128(29)$ \\
\hline Best response of MMR or better to most recent regimen containing dasatinib or nilotinib, $n(\%)^{c}$ & $8(3)$ & $16(4)$ \\
\hline \multicolumn{3}{|l|}{ Baseline cardiovascular risk factors ${ }^{d}$} \\
\hline Arterial hypertension & NA & $240(53)$ \\
\hline Hypercholesterolemia & NA & $219(49)$ \\
\hline Obesity & NA & $109(24)$ \\
\hline Diabetes mellitus & NA & $72(16)$ \\
\hline \multicolumn{3}{|l|}{ Baseline history of cardiovascular disease } \\
\hline Non-ischemic cardiac disease & NA & $193(43)$ \\
\hline Ischemic disease & NA & $102(23)$ \\
\hline \multicolumn{3}{|l|}{ Patient disposition at end of study } \\
\hline Median duration of treatment, mo (range) & $32.1(0.1-73.0)$ & $16.7(0.03-73.0)$ \\
\hline Median follow-up, mo (range) & $56.8(0.1-73.1)$ & $37.3(0.1-73.1)$ \\
\hline Median dose intensity, mg/d (range) & $27.2(5-45)$ & ND \\
\hline \multicolumn{3}{|l|}{ Primary reason for discontinuation, $n(\%)$} \\
\hline Disease progression & $29(11)$ & $105(23)$ \\
\hline Adverse event & $57(21)$ & $79(18)$ \\
\hline Patient request & $31(11)$ & $42(9)$ \\
\hline Lack of efficacy & $15(6)$ & $26(6)$ \\
\hline Death $^{\mathrm{e}}$ & $9(3)$ & $26(6)$ \\
\hline Investigator decision & $11(4)$ & $17(4)$ \\
\hline Lost to follow-up & 0 & $3(<1)$ \\
\hline Non-compliance & $3(1)$ & $4(<1)$ \\
\hline Protocol violation & $2(<1)$ & $2(<1)$ \\
\hline Study closure $e^{f}$ & $90(33)$ & $107(24)$ \\
\hline Other ${ }^{f, g}$ & $14(5)$ & $28(6)$ \\
\hline
\end{tabular}

$C M L$ chronic myeloid leukemia, $C P$ chronic phase, $M M R$ major molecular response, ND not determined, $T K I$ tyrosine kinase inhibitor

${ }^{a}$ Approved TKIs were imatinib, nilotinib, dasatinib, and bosutinib. Previous investigational TKls received by at least $1 \%$ of patients included radotinib (received by $2 \%$ of patients), bafetinib (2\%), rebastinib (2\%), and XL-228 (2\%)

${ }^{\mathrm{b}}$ Assessed by conventional Sanger sequencing at baseline

' Percentages were calculated according to the number of patients who received previous dasatinib or nilotinib: 256 patients with CP-CML, 80 patients with $\mathrm{AP}-\mathrm{CML}$, 61 patients with BP-CML, and 30 patients with $\mathrm{Ph}+\mathrm{ALL}$

d Smoking and family history were not collected as part of the trial. Patients with significant or active cardiovascular disease, including myocardial infarction, unstable angina or congestive heart failure (in prior 3 months), or history of clinically significant atrial or ventricular arrhythmia were excluded from the trial

e Seven deaths were assessed by investigators as possibly or probably related to ponatinib (CP-CML: pneumonia, acute myocardial infarction; AP-CML: fungal pneumonia, gastrointestinal hemorrhage; BP-CML: hemorrhagic gastritis; Ph+ ALL: cardiac arrest, mesenteric arterial occlusion)

${ }^{f}$ Patients who continued to derive clinical benefit from their treatment had the option to receive ponatinib through alternative mechanisms 9 This category includes stem cell transplantation (in 11 patients with CP-CML, 5 with AP-CML, 6 with BP-CML, and 1 with Ph+ ALL). The 9 CP-CML patients and 1 $\mathrm{AP}-\mathrm{CML}$ patient who remained on study at the time of last response assessment are not included in this category. ${ }^{3}$ 


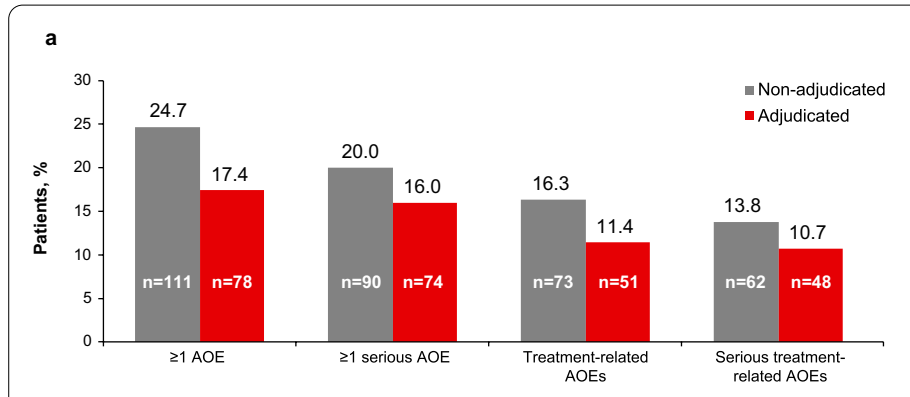

c

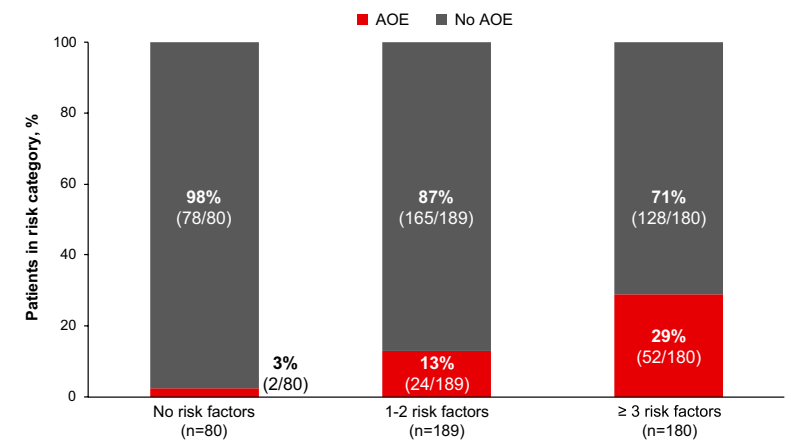

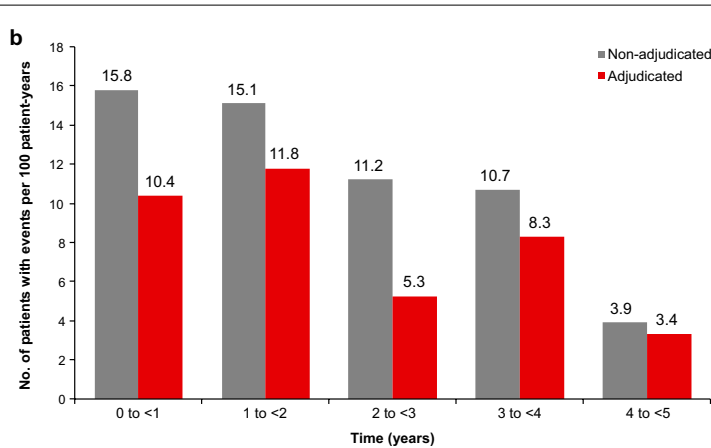

d

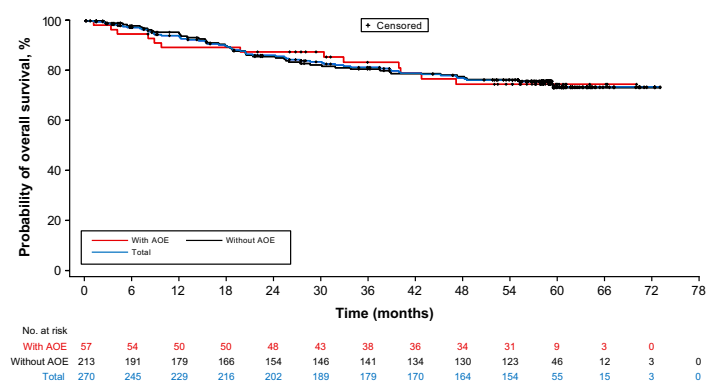

Fig. 2 Arterial occlusive event (AOE) rates with ponatinib. A Rates of non-adjudicated and adjudicated AOEs. B Exposure-adjusted incidence of newly occurring arterial occlusive events (AOEs) by year (all patients). Later intervals excluded patients with prior events. Non-adjudicated values were published previously [3]. C Incidence of AOEs (adjudicated) by number of baseline risk factors (all patients). Risk factors included arterial hypertension, hypercholesterolemia, obesity, diabetes mellitus, non-ischemic cardiac disease, and ischemic disease. D Overall survival (OS) in chronic-phase chronic myeloid leukemia (CP-CML) patients with and without AOEs

with adjudicated AOEs was similar to survival of patients without AOEs (Fig. 2D).

\section{Discussion}

In this study, adjudication of AOEs by an independent committee of experts allowed for a clinically meaningful description of AOEs associated with ponatinib, which can help to inform health care providers and patients of safety risks in an accurate and objective manner. The search that identified potential AOEs for adjudication was broader (based on 604 MedDRA terms related to vascular ischemia or thrombosis) than that initially used to calculate non-adjudicated AOE rates in the PACE trial (400 MedDRA terms) [3]. Based on 5-year followup of the PACE trial, the adjudicated AOE rate (17\%) was lower than the non-adjudicated AOE rate (25\%) [3]. Although the majority of adjudicated AOEs were serious, $81 \%$ of patients with AOEs continued on ponatinib (35\% with dose modifications), the benefit of the drug was felt to outweigh the risk of the AOEs. Although vascular occlusive events were rarely reported during the initial development of second-generation BCR::ABL1 TKIs, a meta-analysis found that these events occurred in $5.9 \%$ of patients with CML treated with these agents, including bosutinib, dasatinib, nilotinib, and ponatinib [13]. In another review of prospective trials of patients treated with TKIs, including imatinib, nilotinib, dasatinib, and ponatinib, overall incidence of $\mathrm{CV}$ events was 45\% (range, 41-63\%) [14]. Accordingly, a high level of vigilance is indicated to recognize this potential complication of TKI therapy.

Notably, although concern existed around the potential for increasing AOE rates with long-term dosing, as seen with AEs related to other TKIs [15-18] the exposureadjusted incidence of newly occurring adjudicated AOEs decreased over time on ponatinib, suggesting that the toxicity of ponatinib may not increase with longer treatment duration.

Patients with adjudicated AOEs were more likely to have multiple baseline cardiovascular risk factors (e.g., ischemic cardiac disease, arterial hypertension, 
Table 5 Rates of non-adjudicated and adjudicated AOEs by type in the total population $(n=449)$

\begin{tabular}{|c|c|c|c|c|}
\hline \multirow[t]{2}{*}{ AOE } & \multicolumn{2}{|c|}{ Non-adjudicated events ${ }^{a}$} & \multicolumn{2}{|c|}{ Adjudicated events $^{b}$} \\
\hline & Any & Serious & Any & Serious \\
\hline Any, & $111(25)$ & $90(20)$ & $78(17)$ & $74(16)$ \\
\hline Cardiovascular ${ }^{c}$ & $59(13)$ & $44(10)$ & $38(8)$ & $37(8)$ \\
\hline \multicolumn{5}{|l|}{ Cardiovascular AOEs in $\geq 1 \%$ of patients } \\
\hline Angina pectoris & $28(6)$ & $15(3)$ & 0 & 0 \\
\hline Acute $\mathrm{Ml}^{\mathrm{d}}$ & $18(4)$ & $18(4)$ & $8(2)$ & $8(2)$ \\
\hline $\mathrm{Ml}$ & d & d & $10(2)$ & $10(2)$ \\
\hline Coronary artery disease & $14(3)$ & $12(3)$ & $7(2)$ & $7(2)$ \\
\hline Acute coronary syndrome & $7(2)$ & $7(2)$ & $7(2)$ & $7(2)$ \\
\hline Coronary artery occlusion & $5(1)$ & $4(1)$ & 0 & 0 \\
\hline Cerebrovascular & $41(9)$ & $33(7)$ & $28(6)$ & $25(6)$ \\
\hline \multicolumn{5}{|l|}{ Cerebrovascular AOEs in $\geq 1 \%$ of patients } \\
\hline Cerebrovascular accident & $11(2)$ & $11(2)$ & $7(2)$ & $7(2)$ \\
\hline Cerebral infarction & $8(2)$ & $8(2)$ & $8(2)$ & $8(2)$ \\
\hline Carotid artery stenosis & $7(2)$ & $6(1)$ & $7(2)$ & $5(1)$ \\
\hline Transient ischemic attack & $6(1)$ & $4(1)$ & 0 & 0 \\
\hline Peripheral vascular & $48(11)$ & $38(8)$ & $42(9)$ & $34(8)$ \\
\hline \multicolumn{5}{|l|}{ Peripheral vascular AOEs in $\geq 1 \%$ of patients } \\
\hline Peripheral arterial occlusive disease & $22(5)$ & $17(4)$ & $19(4)$ & $16(4)$ \\
\hline Intermittent claudication & $11(2)$ & $1(<1)$ & 0 & 0 \\
\hline Peripheral artery stenosis & $10(2)$ & $8(2)$ & $8(2)$ & $7(2)$ \\
\hline Peripheral artery occlusion & $7(2)$ & $5(1)$ & $7(2)$ & $5(1)$ \\
\hline Peripheral ischemia & $7(2)$ & $4(1)$ & $5(1)$ & 0 \\
\hline Peripheral vascular disorder & $5(1)$ & $4(1)$ & 0 & 0 \\
\hline $\begin{array}{l}\text { Exposure-adjusted newly occurring AOEs, patients with } \\
\text { events per } 100 \text { patient-years }\end{array}$ & 13.8 & 10.6 & 8.9 & 8.4 \\
\hline
\end{tabular}

Data are no. (\%) of patients, unless otherwise specified

$A O E$ arterial occlusive event, $C P-C M L$ chronic-phase chronic myeloid leukemia, MedDRA Medical Dictionary for Regulatory Activities, MI myocardial infarction, PT preferred term

${ }^{\text {a }}$ Categorization of AOEs is based on $>400$ MedDRA preferred terms related to vascular ischemia or thrombosis

${ }^{b}$ Events that were adjudicated as an AOE by the adjudication committee

${ }^{\mathrm{C}}$ Does not include arterial hypertension AEs

${ }^{\mathrm{d}}$ Acute $\mathrm{Ml}$ and $\mathrm{MI}$ were grouped as a single category in the non-adjudicated analysis

hypercholesterolemia, and diabetes mellitus), and only 2 patients had an adjudicated AOE without any cardiovascular risk factors. These observations align with those of previous studies $[6,19]$. It is important to identify and manage cardiovascular risk factors before and during therapy with ponatinib or other TKIs [20-22]. In PACE, $80 \%$ of CP-CML patients were resistant to dasatinib or nilotinib, and $24 \%$ had the $B C R:: A B L 1^{\text {T315I }}$ resistance mutation [3]. Among CP-CML patients, estimated 5 -year PFS and OS rates were $53 \%$ and $73 \%$, respectively [3]. Data for overall survival in patients with and without adjudicated AOEs suggest that the risk of AOE-related death did not substantially impact survival, with diseaserelated death being the main driver of the OS curve. This underscores the need for providers to fully understand the therapeutic profile of ponatinib and consider its use when the potential benefits outweigh the risks for a given patient.

This study reinforces the importance of proper assessment of cardiovascular AEs to ensure accurate estimation of cardiovascular risk. The conventional processes of $\mathrm{AE}$ reporting and causality assessment 
Table 6 Rates of AOEs non-adjudicated and adjudicated AOEs in CP-CML patients $(n=270)$

\begin{tabular}{|c|c|c|c|c|}
\hline \multirow[t]{2}{*}{ AOE } & \multicolumn{2}{|c|}{$\begin{array}{l}\text { Non- } \\
\text { adjudicated } \\
\text { events }^{\mathrm{a}}\end{array}$} & \multicolumn{2}{|c|}{$\begin{array}{l}\text { Adjudicated } \\
\text { events }^{\mathrm{b}}\end{array}$} \\
\hline & Any & Serious & Any & Serious \\
\hline Any, & $84(31)$ & $69(26)$ & $57(21)$ & $54(20)$ \\
\hline Cardiovascular ${ }^{\mathrm{c}}$ & $42(16)$ & $33(12)$ & $26(10)$ & $25(9)$ \\
\hline Cerebrovascular & $35(13)$ & $28(10)$ & $25(9)$ & $22(8)$ \\
\hline Peripheral vascular & $38(14)$ & $31(11)$ & $31(11)$ & $26(10)$ \\
\hline $\begin{array}{l}\text { Exposure-adjusted newly occurring } \\
\text { AOEs, patients with events per } 100 \\
\text { patient-years }\end{array}$ & 11.3 & 9.3 & 8.7 & 8.1 \\
\hline
\end{tabular}

Data are no. (\%) of patients, unless otherwise specified

$A O E$ arterial occlusive event, $C P$-CML chronic-phase chronic myeloid leukemia, MedDRA Medical Dictionary for Regulatory Activities, MI myocardial infarction, PT preferred term

${ }^{a}$ Categorization of AOEs is based on $>400$ MedDRA preferred terms related to vascular ischemia or thrombosis

${ }^{\mathrm{b}}$ Events that were adjudicated as an AOE by the adjudication committee

${ }^{\mathrm{C}}$ Does not include arterial hypertension AEs may need to be re-assessed to avoid pitfalls associated with over- or under-reporting of AOEs, both of which may adversely affect patient care [23, 24]. Formal adjudication of events is a mainstay for development programs in other therapeutic areas such as diabetes mellitus $[25,26]$ and cardiology. A better understanding of the AOE risk associated with TKI therapy is a prime example of where formal adjudication is critical because accurate knowledge of risks is crucial before prescribing any TKI. The potential benefits of effective BCR::ABL1 TKI treatment, even with accompanying AEs, may outweigh the potential risks of progression-related mortality in patients with CP-CML and Ph+ ALL receiving second- or thirdline therapy. This is particularly true for patients such as those with the $B C R:: A B L 1^{T 315 I}$ mutation who may have limited treatment options [27]. Understanding the true incidence of the most significant events is a central element in properly assessing the benefit-risk ratio of an intervention. All later-generation TKIs are

Table 7 Arterial occlusive events (AOEs) in $\geq 2.0 \%$ of patients $(n=449)$

\begin{tabular}{|c|c|c|c|c|}
\hline \multirow[t]{2}{*}{ AOE } & \multicolumn{2}{|l|}{ Any AOE } & \multicolumn{2}{|l|}{ Serious AOE } \\
\hline & Non-adjudicated ${ }^{a}$ & Adjudicated $^{\mathrm{b}}$ & Non-adjudicated $^{a}$ & Adjudicated $^{\mathrm{b}}$ \\
\hline Angina pectoris & $28(6)$ & 0 & $15(3)$ & 0 \\
\hline Peripheral arterial occlusive disease & $22(5)$ & $19(4)$ & $17(4)$ & $16(4)$ \\
\hline Myocardial infarction & $18(4)$ & $10(2)$ & $18(4)$ & $10(2)$ \\
\hline Coronary artery disease & $14(3)$ & $7(2)$ & $12(3)$ & $7(2)$ \\
\hline Cerebrovascular accident & $11(2)$ & $7(2)$ & $11(2)$ & $7(2)$ \\
\hline Intermittent claudication & $11(2)$ & 0 & $1(<1)$ & 0 \\
\hline Peripheral artery stenosis & $10(2)$ & $8(2)$ & $8(2)$ & $7(2)$ \\
\hline Cerebral infarction & $8(2)$ & $8(2)$ & $8(2)$ & $8(2)$ \\
\hline Acute coronary syndrome & $7(2)$ & $7(2)$ & $7(2)$ & $7(2)$ \\
\hline Carotid artery stenosis & $7(2)$ & $7(2)$ & $6(1)$ & $5(1)$ \\
\hline Peripheral artery occlusion & $7(2)$ & $7(2)$ & $5(1)$ & $5(1)$ \\
\hline Peripheral ischemia & $7(2)$ & $5(1)$ & $4(1)$ & 0 \\
\hline
\end{tabular}

Data are no. (\%) of patients

MedDRA Medical Dictionary for Regulatory Activities

${ }^{\text {a }}$ Categorization of AOEs is based on MedDRA preferred terms related to vascular ischemia or thrombosis

${ }^{b}$ Events adjudicated as AOEs by the cardiovascular endpoint Adjudication Committee 
Table 8 Time to onset of adjudicated AOEs

\begin{tabular}{lll}
\hline & \multicolumn{2}{l}{$\begin{array}{l}\text { Median time to first AOE (range), } \\
\text { months }\end{array}$} \\
\cline { 2 - 3 } & CP-CML patients & All patients \\
\hline Any AOE & $(n=57)$ & $(n=78)$ \\
& $16.3(0.4,49.5)$ & $14.1(0.1,49.5)$ \\
Cardiovascular AOE & $(n=26)$ & $(n=38)$ \\
Cerebrovascular AOE & $14.1(0.6,52.9)$ & $12.3(0.3,52.9)$ \\
Peripheral vascular AOE & $(n=25)$ & $(n=28)$ \\
& $23.0(0.4,53.5)$ & $18.9(0.4,53.5)$ \\
& $(n=31)$ & $(n=42)$ \\
& $24.6(1.8,49.5)$ & $22.2(0.1,49.5)$ \\
\hline
\end{tabular}

associated with risk of cardiovascular AEs [28], and the results of the formal adjudication process suggest the risk of these events with ponatinib may not be dissimilar to the event rates seen with some second-generation BCR::ABL1 TKIs [16-18].

A noteworthy finding in our analysis is that the exposure-adjusted incidence of newly occurring adjudicated AOEs decreased over time on ponatinib. These results are reassuring that the rate of new AOEs may not increase with longer duration of ponatinib treatment. Furthermore, patients with positively adjudicated AOEs were much more likely to have baseline

Table 9 Ponatinib dose modifications following non-adjudicated and adjudicated arterial occlusive events (AOEs) ${ }^{\text {a }}$

\begin{tabular}{|c|c|c|c|c|}
\hline & \multicolumn{2}{|l|}{ Any AOE } & \multicolumn{2}{|l|}{ Serious AOE } \\
\hline & $\begin{array}{l}\text { Non-adjudicated } \\
(n=111)\end{array}$ & $\begin{array}{l}\text { Adjudicated } \\
(n=78)\end{array}$ & $\begin{array}{l}\text { Non-adjudicated } \\
(n=90)\end{array}$ & $\begin{array}{l}\text { Adjudicated }^{c} \\
(n=74)\end{array}$ \\
\hline No dose modification & $46(41)$ & $36(46)$ & $28(31)$ & $31(42)$ \\
\hline Drug interrupted only & $37(33)$ & $25(32)$ & $37(41)$ & $26(35)$ \\
\hline Dose reduced only & $6(5)$ & 0 & $5(6)$ & 0 \\
\hline Dose reduced + drug interrupted & $5(5)$ & $2(3)$ & $4(4)$ & $2(3)$ \\
\hline Drug interrupted + drug withdrawn & 0 & $2(3)$ & 0 & $2(3)$ \\
\hline Drug withdrawn & $17(15)$ & $5(6)$ & $16(18)$ & $5(7)$ \\
\hline Not applicable/unknown & 0 & $8(10)$ & 0 & $8(11)$ \\
\hline
\end{tabular}

Data are no. (\%) of patients with an AOE

MedDRA Medical Dictionary for Regulatory Activities

${ }^{a}$ When a patient had multiple events, dose modification was derived as the most severe one across all events with the following severity order (high to low): drug withdrawn, drug reduced plus drug interrupted, drug reduced only, drug interrupted only, no dose modification

${ }^{b}$ Categorization of AOEs is based on MedDRA preferred terms related to vascular ischemia or thrombosis

'Events adjudicated as AOEs by the cardiovascular endpoint Adjudication Committee

Table 10 Prevalence of baseline risk factors by adjudicated AOE and serious AOE status

\begin{tabular}{|c|c|c|c|c|}
\hline \multirow[t]{2}{*}{ No. (\%) of patients } & \multicolumn{2}{|l|}{ Any AOE } & \multicolumn{2}{|c|}{ Any serious AOE } \\
\hline & No $(n=371)$ & Yes $(n=78)$ & No $(n=375)$ & Yes $(n=74)$ \\
\hline Age, $\geq 65$ years & $118(32)$ & $37(47)$ & $120(32)$ & $35(47)$ \\
\hline Sex, male & $187(50)$ & $51(65)$ & $188(50)$ & $50(68)$ \\
\hline History of ischemic disease & $45(12)$ & $22(28)$ & $45(12)$ & $22(30)$ \\
\hline Diabetes mellitus & $45(12)$ & $27(35)$ & $48(13)$ & $24(32)$ \\
\hline Baseline glucose grade $\geq 2$ & $24(6)$ & $14(18)$ & $25(7)$ & $13(18)$ \\
\hline Venous thromboembolism & $30(8)$ & $8(10)$ & $30(8)$ & $8(11)$ \\
\hline Arterial hypertension & $181(49)$ & $59(76)$ & $185(49)$ & $55(74.3)$ \\
\hline Baseline blood pressure grade $\geq 2$ & $32(9)$ & $7(9)$ & $32(9)$ & $7(9)$ \\
\hline Hypercholesterolemia & $167(45)$ & $52(67)$ & $169(45)$ & $50(68)$ \\
\hline Baseline triglycerides grade $\geq 1$ & $112(30)$ & $28(36)$ & $114(30)$ & $26(35)$ \\
\hline History of non-ischemic cardiac disease & $120(32)$ & $30(38)$ & $121(32)$ & $29(39)$ \\
\hline Obesity & $88(24)$ & $21(27)$ & $90(24)$ & $19(26)$ \\
\hline Baseline $\mathrm{BMI} \geq 30 \mathrm{~kg} / \mathrm{m}^{-2}$ & $86(23)$ & $21(27)$ & $88(23)$ & $19(26)$ \\
\hline
\end{tabular}

$A O E$ arterial occlusive event, $B M I$ body mass index 
Table 11 Concomitant medication use by adjudicated AOE and serious AOE status

\begin{tabular}{|c|c|c|c|c|}
\hline & Total $(n=449)$ & No AOE $(n=371)$ & Any AOE $(n=78)$ & $\begin{array}{l}\text { Serious } \\
\text { AOE } \\
(n=74)\end{array}$ \\
\hline \multicolumn{5}{|l|}{ Baseline concomitant medications } \\
\hline Antihypertensives & $86(19)$ & $63(17)$ & $23(29)$ & $22(30)$ \\
\hline Acetylsalicylic acid & $39(9)$ & $23(6)$ & $16(21)$ & $15(20)$ \\
\hline Platelet aggregation inhibitors & $38(8)$ & $22(6)$ & $16(21)$ & $15(20)$ \\
\hline Anti-diabetic agents & $24(5)$ & $13(4)$ & $11(14)$ & $10(14)$ \\
\hline Lipid-modifying agents & $22(5)$ & $16(4)$ & $6(8)$ & $6(8)$ \\
\hline Anticoagulants & $15(3)$ & $13(4)$ & $2(3)$ & $2(3)$ \\
\hline \multicolumn{5}{|c|}{ Concomitant medication use at any time } \\
\hline Antihypertensives & $233(52)$ & $181(49)$ & $52(67)$ & $50(68)$ \\
\hline Acetylsalicylic acid & $125(28)$ & $92(25)$ & $33(42)$ & $33(45)$ \\
\hline Platelet aggregation inhibitors & $122(27)$ & $85(23)$ & $37(47)$ & $37(50)$ \\
\hline Anticoagulants & $58(13)$ & $50(13)$ & $8(10)$ & $8(11)$ \\
\hline Lipid-modifying agents & $51(11)$ & $39(11)$ & $12(15)$ & $12(16)$ \\
\hline Anti-diabetic agents & $45(10)$ & $26(7)$ & $19(24)$ & $18(24)$ \\
\hline
\end{tabular}

Data are no. (\%) of patients

$A O E$ arterial occlusive event

cardiovascular risk factors (e.g., arterial hypertension, hypercholesterolemia, diabetes mellitus) or established cardiovascular disease; of those patients without any cardiovascular risk factors only 2 had a subsequent AOE. These results may provide clinical guidance with respect to the approach to use of ponatinib in patients at risk for an AOE. The ongoing phase 2 OPTIC trial (ClinicalTrials.gov Identifier: NCT02467270) is using a response-based dose reduction protocol approach to evaluate the optimal ponatinib dosing regimen for maximizing efficacy while mitigating toxicity. Results show that higher doses of ponatinib were associated with increased incidence of AOEs, with exposure-adjusted treatment-emergent AOE rates of 5.6\%, 3.6\%, and $2.1 \%$ for the 45-mg, 30-mg, and 15-mg cohorts, respectively [29]. However, the benefit differential was considerably larger with a starting dose of $45 \mathrm{mg}$, which was associated with a 26.3 percentage-point improvement in the response rate compared with a 15-mg starting dose (51.6\% vs. $25.3 \%)$ [29]. Overall, the study indicated the best risk/benefit ratio when the $45-\mathrm{mg}$ starting dose was reduced to $15 \mathrm{mg}$ upon achievement of response (BCR::ABL1 $1^{I S}$ transcript levels $\left.\leq 1 \%\right)$ [29].

This retrospective study has strengths and limitations. The adjudication methodology provided a comprehensive and objective approach for characterizing AOE risk. A limitation is that only data from the clinical trial database were available. Prospective implementation of this strategy, as is being done in 2 ongoing trials, OPTIC and Ponatinib-3001 (NCT03589326), will overcome this challenge and add further value to the methodology and strength to the conclusions. In OPTIC, an independent cardiovascular endpoint adjudication committee is reviewing AOEs as they are reported using source documentation including cardiovascular workup (e.g., echocardiograms, electrocardiograms, biomarkers), hospitalization records, and any cardiovascular examinations performed.

\section{Conclusions}

Independent reconsideration of AOEs by an expert adjudication committee showed lower rates of clinically relevant AOEs overall (17\% vs. $25 \%)$ and serious AOEs ( $16 \%$ vs. $20 \%)$ than were originally reported in the PACE trial, suggesting an earlier possible overestimation that may not accurately reflect the AOE risk with ponatinib. The incidence of exposure-adjusted newly occurring AOEs decreased over time during ponatinib treatment. Improved understanding of the AOE profile with ponatinib and risk factors for AOEs can help guide decisions around TKI treatment. Results from the OPTIC study support a novel ponatinib treatment regimen of a $45-\mathrm{mg}$ starting dose reduced to $15 \mathrm{mg}$ upon achievement of response, maximizing response while minimizing toxicity [29]. 
Table 12 Fatal AOEs and patient baseline characteristics

\begin{tabular}{|c|c|c|c|c|c|}
\hline Fatal event & Fatal PT & Other AOE PTs reported & CML/ALL status & History of CV events & $\begin{array}{l}\text { CV risk factors at } \\
\text { baseline }\end{array}$ \\
\hline Bradycardiac arrest & Cardiac arrest & $\begin{array}{l}\text { Cardiac arrest } \\
\text { Dry gangrene } \\
\text { Peripheral ischemia }\end{array}$ & CML & $\begin{array}{l}\text { Congestive heart failure } \\
\text { Hypertension } \\
\text { Impaired diastolic filling } \\
\text { pattern } \\
\text { Left atrium enlargement } \\
\text { Mild tricuspid regurgita- } \\
\text { tion } \\
\text { Mitral valve calcification } \\
\text { without } \\
\text { significant mitral stenosis } \\
\text { Intermittent ventricular } \\
\text { tachycardia }\end{array}$ & $\begin{array}{l}\text { Obesity } \\
\text { Diabetes mellitus } \\
\text { Arterial hypertension }\end{array}$ \\
\hline Cardiac failure & Cardiac failure & $\begin{array}{l}\text { Myocardial infarction } \\
\text { Coronary artery disease } \\
\text { Pulmonary embolism }\end{array}$ & $\mathrm{CML}$ & $\begin{array}{l}\text { Pericarditis } \\
\text { Ischemic heart failure }\end{array}$ & \\
\hline Intracranial hemorrhage & Hemorrhage intracranial & & $\mathrm{CML}$ & $\begin{array}{l}\text { Aortic stenosis } \\
\text { Calcified mitral annulus }\end{array}$ & \\
\hline $\begin{array}{l}\text { Worsening of congestive } \\
\text { heart failure }\end{array}$ & Cardiac failure congestive & $\begin{array}{l}\text { Myocardial infarction } \\
\text { Deep vein thrombosis }\end{array}$ & & $\begin{array}{l}\text { QTc prolongation with } \\
\text { nilotinib use } \\
\text { Stent placement } \\
\text { Congestive heart failure } \\
\text { Myocardial infarction } \\
\text { Coronary artery disease } \\
\text { Mitral regurgitation } \\
\text { Trace of tricuspid valve } \\
\text { regurgitation }\end{array}$ & $\begin{array}{l}\text { Hyperlipidemia } \\
\text { Arterial hypertension }\end{array}$ \\
\hline $\begin{array}{l}\text { Superior mesenteric artery } \\
\text { occlusion }\end{array}$ & $\begin{array}{l}\text { Mesenteric arterial occlu- } \\
\text { sion }\end{array}$ & Celiac artery occlusion & ALL & $\begin{array}{l}\text { Paroxysmal atrial fibril- } \\
\text { lation } \\
\text { Thrombophlebitis } \\
\text { Bilateral leg deep vein } \\
\text { thrombosis } \\
\text { Cardiac catheterization }\end{array}$ & $\begin{array}{l}\text { Hyperlipidemia } \\
\text { Arterial hypertension }\end{array}$ \\
\hline Cardiac arrest & Cardiac arrest & $\begin{array}{l}\text { Peripheral vascular } \\
\text { disorder }\end{array}$ & $\mathrm{ALL}$ & $\begin{array}{l}\text { Greater saphenous vein } \\
\text { thrombosis and cellulitis } \\
\text { Aortic valve slightly } \\
\text { thickened } \\
\text { Left axis deviation } \\
\text { Left bundle branch block } \\
\text { Hypertension } \\
\text { Mild aortic regurgitation } \\
\text { Mild pulmonic valve } \\
\text { regurgitation } \\
\text { Mild to moderate tricus- } \\
\text { pid regurgitation }\end{array}$ & Arterial hypertension \\
\hline $\begin{array}{l}\text { Hemorrhagic cerebral } \\
\text { infarction }\end{array}$ & $\begin{array}{l}\text { Hemorrhagic cerebral } \\
\text { infarction }\end{array}$ & $\begin{array}{l}\text { Cerebral artery stenosis ( } 2 \\
\text { events) } \\
\text { Cerebral infarction ( } 2 \\
\text { events) }\end{array}$ & CML & & $\begin{array}{l}\text { Diabetes mellitus } \\
\text { Arterial hypertension }\end{array}$ \\
\hline Cardiac arrest & Cardiac arrest & & $\mathrm{CML}$ & & \\
\hline Cardiac arrest & Cardiac arrest & & CML & $\begin{array}{l}\text { Ischemic heart disease } \\
\text { Angina pectoris }\end{array}$ & $\begin{array}{l}\text { Coronary artery disease } \\
\text { Type } 2 \text { diabetes mellitus } \\
\text { Hypertension }\end{array}$ \\
\hline Congestive heart failure & Cardiac failure congestive & & $\mathrm{CML}$ & & \\
\hline Stroke & Cerebrovascular accident & $\begin{array}{l}\text { Acute myocardial infarc- } \\
\text { tion ( } 2 \text { events) }\end{array}$ & $C M L$ & $\begin{array}{l}\text { Ischemic stroke } \\
\text { Ischemic heart disease } \\
\text { Coronary artery disease } \\
\text { Revascularization and } \\
\text { coronary stent placement }\end{array}$ & $\begin{array}{l}\text { Diabetes mellitus } \\
\text { Arterial hypertension } \\
\text { Hypercholesterolemia }\end{array}$ \\
\hline
\end{tabular}




\section{Abbreviations}

ACC: American College of Cardiology; AE: Adverse events; AHA: American Heart Association; ALL: Acute lymphoblastic leukemia; AOE: Arterial occlusive event; AP: Accelerated-phase; BP: Blast-phase; CML: Chronic myeloid leukemia; CP-CML: Chronic-phase chronic myeloid leukemia; MeDRA: Medical Dictionary for Regulatory Activities; PACE: Ponatinib Ph+ ALL and CML Evaluation; PFS: Progression-free survival; Ph+: Philadelphia chromosome positive; qd: Once daily; SCTI: Standardized Data Collection for Cardiovascular Trials Initiative; SMQ: Cardiac Failure Standard MedDRA Query; TKI: Tyrosine kinase inhibitor.

\section{Acknowledgements}

We thank the patients, their families, and their caregivers, and the study investigators and their team members at each site for participation in the PACE trial. Professional medical writing assistance was provided by Peloton Advantage, LLC, an OPEN Health company, Parsippany, NJ, USA, and funded by Millennium Pharmaceuticals, Inc., Cambridge, MA, USA, a wholly owned subsidiary of Takeda Pharmaceutical Company Limited.

Presented in part at Januzzi, J.L., Garasic, J., Kasner, S. et al. (2020). "An independent review of arterial occlusive events (AOEs) in the ponatinib (PON) phase II PACE trial (NCT01207440) in patients (pts) with Ph+ leukemia [abstract]." Journal of Clinical Oncology 38(15 suppl): 7550. Januzzi, J., Garasic, J., Kasner, S. et al. (2020). "Retrospective independent review of arterial occlusive events (AOES) in the phase 2 pace trial of ponatinib in Philadelphia chromosome-positive $(\mathrm{PH}+$ ) leukemia [abstract]." HemaSphere 4(Suppl 1): 338; Januzzi, J., Garasic, J., Kasner, S. et al. (2020). "Retrospective independent review of arterial occlusive events in the phase 2 pace trial of ponatinib in Philadelphia chromosome-positive leukemia [abstract]." Presented at the 8th Annual Meeting (Virtual) of the Society of Hematologic Oncology ( $\mathrm{SOHO}$ ), September 9-12, 2020; Januzzi, J., Garasic, J., Kasner, S. et al. (2020). "Retrospective independent review of arterial occlusive events in the phase 2 pace trial (NCT01207440) of ponatinib in Philadelphia chromosome-positive leukemia [abstract]." Presented at the John Goldman E-Conference on Chronic Myeloid Leukemia: Biology and Therapy (iCMLf) October 1-4, 2020.xxx

\section{Authors' contributions}

$K C, E B, D N, J X, S S, J L, T H$, and JC were involved in the conception and design. $J C, M M, M D, A H, J P-I, F N, D-W K, D J D$, and HK contributed to the provision of study material or patients. JMG, SEK, VM, MCP, JC, MM, MD, AH, JP-I, FN, D-WK, DJD, HK, SS, TH, JX, and DN contributed to the collection and assembly of data. All authors contributed to the data analysis and interpretation. All authors wrote the manuscript. All authors were involved in the final approval of manuscript. All authors are accountable for all aspects of the work. All authors performed data analysis and interpretation, had full access and verified all the data in the study, and had final responsibility for the decision to submit for publication. All authors were involved in drafting and providing critical revision of the article. All authors read and approved the final manuscript.

\section{Funding}

The PACE study is sponsored by ARIAD Pharmaceuticals, Inc., Cambridge, MA, USA, a wholly owned subsidiary of Takeda Pharmaceutical Company Limited.

\section{Availability of data and materials}

The data sets, including the redacted study protocol, redacted statistical analysis plan, and individual participants data supporting the results reported in this article, will be made available within three months from initial request, to researchers who provide a methodologically sound proposal. The data will be provided after its de-identification, in compliance with applicable privacy laws, data protection and requirements for consent and anonymization.

\section{Declarations}

\section{Ethics approval and consent to participate}

PACE was approved by local ethics committees and was conducted in accordance with the Declaration of Helsinki and the International Council for Harmonisation guidelines for good clinical practice. All patients provided written informed consent.

\section{Consent for publication}

All authors have critically reviewed the manuscript and consent to publication.

\section{Competing interests}

J.L.J.: Consulting/advisory role (Takeda), research funding (Novartis); J.M.G.: Employment and stock/other ownership (family member: Vertex Pharmaceuticals), consulting/advisory role (Clinical Events Committee, ACl; AbbVie; Baim Institute; Parexel), research funding (ReCor Medical); S.E.K.: research funding (Bristol Myers Squibb, Genentech, Medtronic), consulting/advisory role (AbbVie, Abbott, AstraZeneca, BMS, Janssen, Takeda, Medtronic); V.M.: Consulting/advisory role (Takeda, Novartis, Amgen, Bayer), honoraria (Amgen, Novartis), research funding (Novartis, Rigel); M.C.P.: Endpoint adjudication committee (Takeda); J.S.: Employment, leadership role, stock/other ownership (WCG Clinical); M.M.: Consulting/advisory role, travel/accommodations/ expenses, and honoraria (Novartis, BMS, Pfizer, Takeda), research funding (all to institution: Sun Pharma, Novartis, BMS); K.C.: Honoraria, consulting/ advisory role, travel/accommodations/expenses (Takeda, Abbott, CSI, Philips, Abiomed, Cordis, Boston Scientific), research funding (Abbott, ARIAD, Takeda); E.B.: Honoraria, consulting/advisory role, travel/accommodations/expenses (ARIAD); MD: Consulting/advisory role (Blueprint, Fusion Pharma, Takeda, Humana, Ascentage Pharma, Adelphi, Medscape, Novartis), research funding (Takeda, Pfizer, Novartis, Incyte, SPARC, Blueprint, Leukemia \& Lymphoma Society); A.H.: Research funding (Incyte, BMS, Novartis, Pfizer); J.P.I.: Consulting/advisory role (AbbVie, Janssen, AstraZeneca, Novartis, TG Therapeutics, Takeda), speakers bureau (AbbVie, Janssen, AstraZeneca, Takeda), research funding (MEl, Sunesis), patents/royalties/other intellectual property (Sellas); F.N.: Honoraria, speakers bureau, travel/accommodations/expenses (Novartis, Incyte Biosciences), consulting/advisory role (Sun Pharma), research funding (Incyte Biosciences); D.W.K.: Research funding (Novartis, BMS, Pfizer, Takeda, Sun Pharma, Il-Yang Pharm. Co., Ltd.), advisory board (Novartis, BMS); D.J.D.: Consulting/advisory role (Incyte, Pfizer, BMS, Amgen, Novartis, Celgene, Immunogen, Takeda, Blueprint Medicines), research funding (all to institution: Novartis, AbbVie, GlycoMimetics, Blueprint Medicines); H.K.: Honoraria (AbbVie, Amgen, ARIAD, BMS, Immunogen, Orsenix, Pfizer, Agios, Takeda, Actinium Pharmaceuticals), research funding (all to institution: Pfizer, Amgen, BMS, Novartis, ARIAD, Astex Pharmaceuticals, AbbVie, Agios, Cyclacel, Immunogen, Jazz Pharmaceuticals); J.X.: Employment (Takeda); T.H.: Employment (Takeda); S.S.: Employment (Takeda); D.N.: Employment (Takeda); J.C.: Consulting/ advisory role (BMS, Novartis, Pfizer, Takeda), research funding (Novartis, Pfizer, Takeda, Sun Pharma).

\section{Author details}

${ }^{1}$ Massachusetts General Hospital, 55 Fruit Street, Boston, MA, USA. ${ }^{2}$ University of Pennsylvania, Philadelphia, PA, USA. ${ }^{3}$ Barts Health NHS Trust, London, England. ${ }^{4}$ University of Glasgow, Glasgow, Scotland. ${ }^{5} \mathrm{ACI}$ Clinical, Bala Cynwyd, PA, USA. ${ }^{6}$ Memorial Sloan Kettering Cancer Center, New York, NY, USA. ${ }^{7}$ Brigham and Women's Hospital, Harvard Medical School, Boston, MA, USA. ${ }^{8}$ Huntsman Cancer Institute, The University of Utah, Salt Lake City, UT, USA. ${ }^{9}$ Universitätsklinikum Jena, Jena, Germany. ${ }^{10} \mathrm{H}$. Lee Moffitt Cancer Center \& Research Institute, Tampa, FL, USA. ${ }^{11}$ Centre Hospitalier Lyon-Sud, Pierre-Bénite, Lyon, France. ${ }^{12}$ Catholic Hematology Hospital, Seoul St. Mary's Hospital, Leukemia Research Institute, The Catholic University of Korea, Seoul, South Korea.

${ }^{13}$ Dana-Farber Cancer Institute, Boston, MA, USA. ${ }^{14}$ The University of Texas MD Anderson Cancer Center, Houston, TX, USA. ${ }^{15}$ Millennium Pharmaceuticals, Inc., A Wholly Owned Subsidiary of Takeda Pharmaceutical Company Limited, Cambridge, MA, USA. ${ }^{16}$ Georgia Cancer Center, Augusta, GA, USA.

Received: 21 September 2021 Accepted: 18 December 2021 Published: 6 January 2022

\section{References}

1. O'Hare T, Shakespeare WC, Zhu X, et al. AP24534, a pan-BCR-ABL inhibitor for chronic myeloid leukemia, potently inhibits the T315I mutant and overcomes mutation-based resistance. Cancer Cell. 2009;16:401-12.

2. Cortes JE, Kim DW, Pinilla-lbarz J, et al. A phase 2 trial of ponatinib in Philadelphia chromosome-positive leukemias. N Engl J Med. 2013;369:1783-96.

3. Cortes JE, Kim DW, Pinilla-lbarz J, et al. Ponatinib efficacy and safety in Philadelphia chromosome-positive leukemia: final 5-year results of the phase 2 PACE trial. Blood. 2018;132:393-404. 
4. Breccia M, Abruzzese E, Castagnetti F, et al. Ponatinib as second-line treatment in chronic phase chronic myeloid leukemia patients in real-life practice. Ann Hematol. 2018;97:1577-80.

5. Shacham-Abulafia A, Raanani P, Lavie D, et al. Real-life experience with ponatinib in chronic myeloid leukemia: a multicenter observational study. Clin Lymphoma Myeloma Leuk. 2018;18:e295-301.

6. Caocci G, Mulas O, Abruzzese $\mathrm{E}$, et al. Arterial occlusive events in chronic myeloid leukemia patients treated with ponatinib in the real-life practice are predicted by the Systematic Coronary Risk Evaluation (SCORE) chart. Hematol Oncol. 2019;37:296-302.

7. Heiblig M, Rea D, Chretien ML, et al. Ponatinib evaluation and safety in real-life chronic myelogenous leukemia patients failing more than two tyrosine kinase inhibitors: the PEARL observational study. Exp Hematol. 2018:67:41-8

8. Ashaye AO, Thomas C, Dalal M, et al. Treatment of newly diagnosed Philadelphia chromosome positive acute lymphoblastic leukemia using tyrosine kinase inhibitors in combination with chemotherapy: a patientcentered benefit-risk assessment [abstract]. Blood. 2020;136(Suppl 1):20-1.

9. Kantarjian HM, Deininger MW, Abruzzese E, et al. Efficacy and safety of ponatinib (PON) in patients with chronic-phase chronic myeloid leukemia (CP-CML) who failed one or more second-generation (2G) tyrosine kinase inhibitors (TKIs): analyses based on PACE and Optic [abstract]. Blood. 2020;136(suppl 1):43-4.

10. Hicks KA, Hung HMJ, Mahaffey KW, et al. Standardized definitions for cardiovascular and stroke endpoint events in clinical trials. 2014. Available at https://www.cdisc.org/system/files/all/standard/Draft\%20Definitions\% 20for\%20CDISC\%20August\%2020\%2C\%202014.pdf. Accessed 14 Nov 2019

11. Hicks KA, Tcheng JE, Bozkurt B, et al. 2014 ACC/AHA key data elements and definitions for cardiovascular endpoint events in clinical trials: a report of the American College of Cardiology/American Heart Association Task Force on Clinical Data Standards (writing committee to develop cardiovascular endpoints data standards). J Am Coll Cardiol. 2015;66:403-69.

12. Hicks KA, Mahaffey KW, Mehran R, et al. 2017 cardiovascular and stroke endpoint definitions for clinical trials. Circulation. 2018;137:961-72.

13. Douxfils J, Haguet H, Mullier F, Chatelain C, Graux C, Dogne JM. Association between BCR-ABL tyrosine kinase inhibitors for chronic myeloid leukemia and cardiovascular events, major molecular response, and overall survival: a systematic review and meta-analysis. JAMA Oncol. 2016;2:625-32.

14. Jain P, Kantarjian H, Boddu PC, et al. Analysis of cardiovascular and arteriothrombotic adverse events in chronic-phase CML patients after frontline TKls. Blood Adv. 2019;3:851-61.

15. Hochhaus A, Saglio G, Hughes TP, et al. Long-term benefits and risks of frontline nilotinib versus imatinib for chronic myeloid leukemia in chronic phase: 5-year update of the randomized ENESTnd trial. Leukemia. 2016:30:1044-54

16. Kantarjian HM, Hughes TP, Larson RA, et al. Long-term outcomes with frontline nilotinib versus imatinib in newly diagnosed chronic myeloid leukemia in chronic phase: ENESTnd 10-year analysis. Leukemia. 2021:35:440-53.

17. Rea D, Mirault T, Raffoux E, et al. Identification of patients (pts) with chronic myeloid leukemia $(\mathrm{CML})$ at high risk of artery occlusive events (AOE) during treatment with the 2nd generation tyrosine kinase inhibitor (TKI) nilotinib, using risk stratification for cardiovascular diseases (CVD) [abstract]. Blood. 2013;122:2726.

18. Valent P, Hadzijusufovic E, Schernthaner GH, Wolf D, Rea D, le Coutre P. Vascular safety issues in CML patients treated with BCR/ABL1 kinase inhibitors. Blood. 2015;125:901-6.

19. Dorer DJ, Knickerbocker RK, Baccarani M, et al. Impact of dose intensity of ponatinib on selected adverse events: multivariate analyses from a pooled population of clinical trial patients. Leuk Res. 2016;48:84-91.

20. Cortes J. How to manage CML patients with comorbidities. Blood. 2020:136:2507-12.

21. Hochhaus A, Baccarani M, Silver RT, et al. European LeukemiaNet 2020 recommendations for treating chronic myeloid leukemia. Leukemia. 2020;34:966-84
22. Steegmann JL, Baccarani M, Breccia M, et al. European LeukemiaNet recommendations for the management and avoidance of adverse events of treatment in chronic myeloid leukaemia. Leukemia. 2016;30:1648-71.

23. Russo Rossi A, Breccia M, Abruzzese E, et al. Outcome of 82 chronic myeloid leukemia patients treated with nilotinib or dasatinib after failure of two prior tyrosine kinase inhibitors. Haematologica. 2013;98:399-403.

24. Ongoren S, Eskazan AE, Suzan V, et al. Third-line treatment with secondgeneration tyrosine kinase inhibitors (dasatinib or nilotinib) in patients with chronic myeloid leukemia after two prior TKIs: real-life data on a single center experience along with the review of the literature. Hematology. 2018;23:212-20.

25. Guidance for Industry Diabetes Mellitus_evaluating cardiovascular risk in new antidiabetic therapies to treat type 2 diabetes. 2008. Available at https://www.fda.gov/media/71297/download. Accessed 1 Apr 2021.

26. US Food and Drug Administration. Type 2 diabetes mellitus: evaluating the safety of new drugs for improving glycemic control; draft guidance for industry; availability. Fed Regist. 2020;85:13903-5.

27. Moslehi JJ, Deininger M. Tyrosine kinase inhibitor-associated cardiovascular toxicity in chronic myeloid leukemia. J Clin Oncol. 2015;33:4210-8.

28. Medeiros BC, Possick J, Fradley M. Cardiovascular, pulmonary, and metabolic toxicities complicating tyrosine kinase inhibitor therapy in chronic myeloid leukemia: strategies for monitoring, detecting, and managing. Blood Rev. 2018:32:289-99.

29. Cortes J, Apperley JF, Lomaia E, et al. Ponatinib dose-ranging study in chronic-phase chronic myeloid leukemia: a randomized, open-label phase 2 clinical trial. Blood. 2021;138:2042-50.

\section{Publisher's Note}

Springer Nature remains neutral with regard to jurisdictional claims in published maps and institutional affiliations. 